\title{
Robust precoder-decoder design for physical layer network coding-based MIMO two-way relaying system
}

\author{
Laddu Keeth Saliya Jayasinghe ${ }^{*}$, Nandana Rajatheva and Matti Latva-Aho
}

\begin{abstract}
In this paper, we investigate a robust joint precoder-decoder design scheme for a multiple-input multiple-output physical layer network coding (PNC)-based two-way relay system. An orthogonal training sequence is used to estimate the channels. The estimate is imperfect, and a robust design is proposed to find precoders at the source nodes and decoder at the relay node to facilitate PNC operations during multiple-access stage. Both channel estimation error and antenna correlations are used to formulate the optimization problem to minimize the weighted mean square error (WMSE) under a total power constraint. The problem becomes non-convex, and we propose an algorithm to solve it optimally. During the broadcast stage, an algorithm is proposed to find a precoder at the relay node and decoders at source nodes. The system performance is evaluated with estimation error and antenna correlation parameters. The effect of weighting parameters, relay location, and number of antennas at nodes are also considered in the numerical analysis. Numerical results confirm that our joint precoder-decoder algorithms provide the optimal solution to the minimization of WMSE with the total available power.
\end{abstract}

Keywords: Bit error rate, Imperfect channel state information, Mean square error, Multiple-input multiple-output, Non-convex optimization, Physical layer network coding

\section{Introduction}

Cooperative relay communication is introduced to improve the throughput, extend the coverage area, and reduce the energy consumption at the transmitter in wireless communication systems. Relaying information through several hops reduces the need to use large power at the transmitter, which in turn results in a lower level of interference [1]. However, even with these advantages, the relay alone has a disadvantage, which is due to half-duplex signaling, i.e., the node cannot transmit and receive signals simultaneously without complicated interference canceling techniques. This reduces the spectral efficiency to a considerable degree.

A significant amount of research effort has been focused on this weakness, and new methods are proposed to overcome the problem. In [2,3], various system models have been studied to provide solutions on this drawback, and

*Correspondence: kladdu@ee.oulu.fi

Centre for Wireless Communications (CWC), Department of Communications Engineering, University of Oulu, Oulu 90570, Finland more suitable schemes are considered in $[4,5]$ with network coding. The authors in [6] proposed a two-way relaying scheme to avoid this problem which requires only two time slots to exchange information between two source nodes as opposed to four in a traditional relaying scheme. In these two-way relaying systems, the first time slot is known as the multiple access (MA) stage, where both source nodes transmit to the relay at the same time. The second time slot is the broadcasting (BC) stage, where the relay broadcasts the message. Different signal processing methods can be used at the relay node. The most common approach is to employ the amplify-forward (AF) technique at the relay. Recently, new approaches have been investigated using physical layer network coding (PNC) [5,7-9]. The PNC treats interference as useful information. Therefore, PNC improves the capacity of the system and overcomes the shortcomings mentioned earlier in the use of relays. In [7,9], the authors performed exclusive-or (XOR) operation at the relay and transmitted a modulated signal version of that during the second time slot. Another approach was used in [8], where the

\section{是 Springer}

(c) 2013 Saliya Jayasinghe et al.; licensee Springer. This is an Open Access article distributed under the terms of the Creative Commons Attribution License (http://creativecommons.org/licenses/by/2.0), which permits unrestricted use, distribution, and reproduction in any medium, provided the original work is properly cited. 
relay estimates the sum of two transmitted messages and forwards it during the next time slot. This is less complex and valid for most modulation schemes, and the carrier phase offset between the signals will not affect the performance. Further studies on PNC-based relaying are discussed in $[5,9,10]$, and it is found that PNC results in rate improvements of wireless relay systems. Multiple-input multiple-output (MIMO) communication is another spectrally efficient technique that can be used to improve the wireless system performance. MIMO systems have significant performance enhancements over the single-input single-output counterpart [11], and they also provide higher spectral efficiency in the presence of multi-path fading channels [12-14].

Both MIMO and PNC are considered together in some recent research efforts and are identified as potential capacity enhancement techniques for future wireless systems. In most of these studies, it is required to find a relation between two transmitted signals to perform PNC mapping at the relay node. Even though the PNC mapping is well established for single antenna scenarios, there are no such models to handle MIMO PNC mapping. The authors in [15-18] have used MIMO two-way relaying schemes and discussed the detection mechanisms and performances of such systems. Most of these need complex processing at the relay to estimate the XOR of two received signal vectors. A precoder before transmission is proposed in [16], and that reduces the complexity of the receiver at the relay node. The MIMO two-way relaying system can then be shown to produce spatial multiplexing streams; hence, the PNC operation at the relay becomes less complex. In [19], power allocation is considered with zero-forcing precoders at the source nodes to reduce the complexity of PNC operation at the relay node.

Moreover, a joint precoder-decoder design can be thought of as an effective way to reduce the inter-datastream interference that lowers the complexity of the PNC operation further by placing signal processing capabilities at both transmitters and the receiver. Previous studies on AF two-way relaying suggest that the joint precoder and decoder design can achieve more effective capacity enhancements and decoding performances [20-22]. The focus is mainly on minimizing the weighted mean square error (WMSE) in an AF two-way relay system. In PNC-based two-way relaying systems, the summation of the transmitted signal vectors is needed to map it to either of the XOR version or to transmit the estimated summation. Therefore, MSE is a good measure to design precoders and decoders in PNC-based two-way relaying.

In most of these related cases, channel state information (CSI) is assumed to be known perfectly in all nodes [19,21-23]. Precoder design at nodes with ideal channel information is not a practical scenario in general. Channel estimation has errors, and it will affect the system performance in a significant manner. Several research studies are carried out by considering various models of imperfect CSI and their impact on the performance of wireless systems. In [24,25], the authors considered an imperfect CSI model with estimation errors in channel parameters, and they used a training sequence to estimate the channel. In [25-27], transceiver designs for AF MIMO relaying systems are discussed based on this imperfect CSI model. The authors in [28] studied the impact of imperfect CSI in a two-way relaying system. However, their work is limited to a single antenna with expressions for bit error rate (BER) of BPSK scheme. Another imperfect CSI model is discussed in [29-31] for a scenario where the channel parameters are estimated at the receiver and fed to the information to the transmitter and relay node. This can cause feedback delays, and the main concern is the difference between the actual CSI and the outdated CSI. However, the feedback delay of CSI estimates has a similar model as for the imperfect estimation. In all these models, consideration of imperfect CSI is very important since focusing only on the perfect information is not sufficient for practical implementations. Having been motivated with these facts, we concentrate our research on robust precoder and decoder designs for MIMO PNC two-way relay system. We also consider the correlation among antennas, which is practical to be assumed in modern compact devices which have sophisticated features. The proposed system design is compared with other alternatives. Similar studies are not considered in the open literature, to the best of our knowledge.

In this paper, our main focus is to design robust precoders and decoders for source and relay nodes to minimize the WMSE. A channel estimation method is proposed for two-way relaying systems using alreadyknown orthogonal sequence transmission techniques. Errors in the estimation are illustrated, and those are considered in the joint precoder-decoder design problems. Both MA and $\mathrm{BC}$ stages are considered in the design of precoders and decoders. Unfortunately, WMSEbased optimization problems become non-convex, and we present methods of solving those by suitable reformulation. Different scenarios are investigated to find the impact of the estimation error, antenna correlation, weighting parameters, relay location, and number of antennas to the design. Performance differences are highlighted, comparing joint precoder-decoder designs with other possibilities.

The rest of the paper is organized as follows. In Section 2, we describe the system model, channel estimation, and the basic problem formulation. In Section 3, we study the optimum design for precoders and the decoder, and Section 4 gives the numerical results. Finally, conclusions are given in Section 5. 
Notations: $\mathbb{C}^{m \times n}$ denotes an $m \times n$ matrix with elements in the complex field. Capital bold letters represent matrices, simple bold letters represent vectors, and simple letters represent scalar variables. $\operatorname{Tr}(\cdot),(\cdot)^{-1},(\cdot)^{T},(\cdot)^{*}$, and $(\cdot)^{H}$ indicate trace, inverse, transpose, conjugate, and hermitian of a matrix, respectively. $\mathcal{E}\{\cdot\}$ and $\Re(z)$ denote the expectation of a random variable and real part of $z$. $\operatorname{vec}(\cdot)$ gives matrix vectorization operator, and $\otimes$ represents the Kronecker operator. $\mathcal{C N}(x, y)$ denotes a complex Gaussian random variable with mean $x$ and variance $y$. A similar notation with mean vector and covariance matrix is valid when the variable is a vector.

\section{System model and problem formulation}

A basic two-way communication system model is considered as shown in Figure 1. The system operates in the time division duplex mode. Source 1 and source 2 are required to exchange data between themselves with the assistance of a relay node. All three nodes have $N$ antennas. The relay is located at a normalized distance $d$ from source 1. Source 1-to-relay and source 2-to-relay transmissions undergo Rayleigh fading with $\mathbf{H}_{1} \in \mathbb{C}^{N \times N}$ and $\mathbf{H}_{2} \in \mathbb{C}^{N \times N}$, respectively. Entries of $\mathbf{H}_{1}$ are assumed to be approximately $\frac{1}{d^{\alpha}} \mathcal{C N}(0,1)$, and entries of $\mathbf{H}_{2}$ are assumed to be approximately $\frac{1}{(2-d)^{\alpha}} \mathcal{C N}(0,1)$, where $\alpha$ is the path loss exponent and $d$ is the normalized distance. The distance between any node to the midpoint of two nodes is considered as the reference distance.

We consider $\mathbf{R}_{i} \in \mathbb{C}^{N \times N}$ as the antenna correlation matrix at source $i(=1,2)$, and $\mathbf{R}_{r} \in \mathbb{C}^{N \times N}$ is the antenna correlation matrix at the relay node. We consider these matrices to be symmetric. We denote $\mathbf{H}_{m 1}=\mathbf{R}_{r}^{\frac{1}{2}} \mathbf{H}_{1} \mathbf{R}_{1}^{\frac{1}{2}}$ and $\mathbf{H}_{m 2}=\mathbf{R}_{r}^{\frac{1}{2}} \mathbf{H}_{2} \mathbf{R}_{2}^{\frac{1}{2}}$ to be the channel matrices from source 1-to-relay and source 2-to-relay. During the BC stage, channel matrices are denoted as $\mathbf{H}_{b 1}=\mathbf{R}_{1}^{\frac{1}{2}} \mathbf{H}_{1}^{T} \mathbf{R}_{r}^{\frac{1}{2}}$ and $\mathbf{H}_{b 2}=\mathbf{R}_{2}^{\frac{1}{2}} \mathbf{H}_{2}^{T} \mathbf{R}_{r}^{\frac{1}{2}}$.
During the MA stage, the source nodes transmit their signals and the summation of two signals are received at the relay node. The relay node estimates the sum of the two signals, which is the general scheme of PNC mapping [8]. However, a problem arises due to channel fading. With fading, the estimation at the relay node becomes exceedingly complex. Therefore, we consider designing precoders at the source nodes and a decoder at the relay node to overcome this problem. Source nodes use precoders $\mathbf{F}_{1} \in \mathbb{C}^{N \times N}$ and $\mathbf{F}_{2} \in \mathbb{C}^{N \times N}$ before their transmissions. The relay node receives both signals at the same time and uses the decoder $\mathbf{G} \in \mathbb{C}^{N \times N}$ prior to performing any PNC operation as in [8] to estimate the XOR or the summation of two transmitted symbol vectors.

Next, we consider the BC stage of two-way communication. The relay node retransmits the estimated summation during this stage. The source nodes estimate the summation at their nodes and find the respective symbol transmitted by the other node with the help of its own symbol. Here, we consider the precoder at the relay node and decoders at the source nodes. The relay uses the precoder $\mathbf{F}_{r} \in \mathbb{C}^{N \times N}$. Both sources 1 and 2 use decoders $\mathbf{G}_{1} \in \mathbb{C}^{N \times N}$ and $\mathbf{G}_{2} \in \mathbb{C}^{N \times N}$, and each reconstructs the required symbol with the help of their own information. All nodes dynamically adjust their precoder and decoder matrices with the channel information.

Both stages require CSI knowledge to design the precoders and the decoder, and the following procedure is used to estimate channels prior to their data transmission.

\subsection{Channel estimation}

We assume channel reciprocity and a quasi-static channel environment. The relay transmits an orthogonal sequence to estimate channels at the end of the $\mathrm{BC}$ time slot. Both source nodes receive this signal, and they find optimum precoder and decoder matrices. The source nodes then send optimum $\mathbf{G}$ and $\mathbf{F}_{r}$ to the relay node via dedicated feedback link. We assume that the channel estimation and

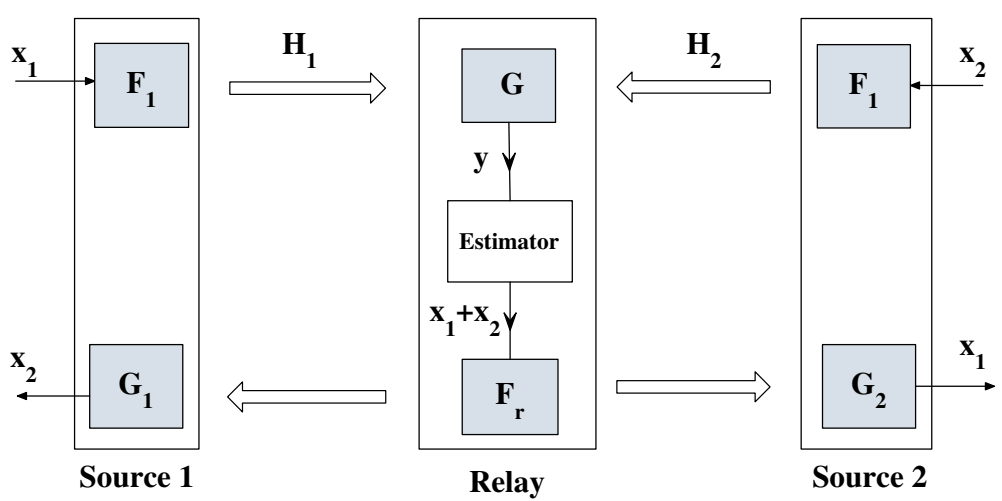

Figure 1 System diagram. System diagram for multiple-access and broadcast stages. Each node consists of $N$ antennas. 
relay feedback time durations are very small compared to the channel coherence time. Once the two-way relaying system is established with optimum precoders and decoders, the source nodes transmit data that are required to exchange between them. Antenna correlations are also assumed to be known.

The relay broadcasts $\mathbf{X}_{t}$ training sequence, where $\mathbf{X}_{t}$ is an $N \times N$ matrix. The received signal at source 1 is given by the following:

$$
\mathbf{Y}_{1}=\mathbf{H}_{b 1} \mathbf{X}_{t}+\mathbf{N}_{1} \text {, }
$$

where $\mathbf{N}_{1}$ is an $N \times N$ matrix with entries independent and identically distributed (i.i.d.) $\mathcal{C N}\left(0, \sigma_{N_{1}}^{2}\right)$. As commonly used in orthogonal sequence channel estimation [24], we use $\mathbf{X}_{t}=\mathbf{R}_{r}^{-\frac{1}{2}} \mathbf{X}$, where $\mathbf{X}$ is a unitary $N \times N$ matrix with multiplication factor $\sqrt{P_{t} / \operatorname{Tr}\left(\mathbf{R}_{r}^{-1}\right)}$ with the relay transmit power $P_{t}$. Source 1 pre-multiplies the received signal matrix by $\mathbf{R}_{1}^{-\frac{1}{2}}$ and post-multiplies it by $\mathbf{X}^{-1}$. Therefore, the received channel matrix $\widetilde{\mathbf{H}}_{1}^{T}$ is given by the following:

$$
\begin{aligned}
\widetilde{\mathbf{H}}_{1}^{T} & =\mathbf{R}_{1}^{-\frac{1}{2}} \mathbf{R}_{1}^{\frac{1}{2}} \mathbf{H}_{1}^{T} \mathbf{R}_{r}^{\frac{1}{2}} \mathbf{R}_{r}^{-\frac{1}{2}} \mathbf{X} \mathbf{X}^{-1}+\mathbf{R}_{1}^{-\frac{1}{2}} \mathbf{N}_{1} \mathbf{X}^{-1} \\
& =\mathbf{H}_{1}^{T}+\mathbf{R}_{1}^{-\frac{1}{2}} \widetilde{\mathbf{N}}_{1},
\end{aligned}
$$

where $\widetilde{\mathbf{N}}_{1}$ is an $N \times N$ matrix with entries i.i.d. $\mathcal{C N}\left(0, \sigma_{1}^{2}\right)$ and $\sigma_{1}^{2}=\sigma_{N_{1}}^{2} \operatorname{Tr}\left(\mathbf{R}_{r}^{-1}\right) / P_{t}$. The minimum MSE (MMSE) criterion is used to obtain the channel estimate from $\widetilde{\mathbf{H}}_{1}^{T}$. The MMSE estimate is presented as follows:

$$
\overline{\mathbf{H}}_{1}^{T}=\mathcal{E}\left\{\mathbf{H}_{1}^{T} \mid \widetilde{\mathbf{H}}_{1}^{T}\right\}=\left[\mathbf{I}_{N}+\sigma_{1}^{2} \mathbf{R}_{1}^{-1}\right]^{-1} \widetilde{\mathbf{H}}_{1}^{T} .
$$

The estimation error can be obtained [27] as $\mathbf{R}_{1}^{-\frac{1}{2}}\left[\mathbf{I}_{N}+\sigma_{1}^{2} \mathbf{R}_{1}^{-1}\right]^{-\frac{1}{2}} \mathbf{E}_{1}$, where $\mathbf{E}_{1}$ is an $N \times N$ matrix with entries i.i.d. $\mathcal{C N}\left(0, \sigma_{1}^{2}\right)$. Therefore, the channel matrix now consists of MMSE estimation and the estimation error part as

$$
\mathbf{H}_{1}^{T}=\overline{\mathbf{H}}_{1}^{T}+\mathbf{R}_{1}^{-\frac{1}{2}}\left[\mathbf{I}_{N}+\sigma_{1}^{2} \mathbf{R}_{1}^{-1}\right]^{-\frac{1}{2}} \mathbf{E}_{1} .
$$

A similar result is valid for the estimation of $\mathbf{H}_{2}^{T}$, and it is given by $\mathbf{H}_{2}^{T}=\overline{\mathbf{H}}_{2}^{T}+\mathbf{R}_{2}^{-\frac{1}{2}}\left[\mathbf{I}_{N}+\sigma_{2}^{2} \mathbf{R}_{2}^{-1}\right]^{-\frac{1}{2}} \mathbf{E}_{2}$, where $\mathbf{E}_{2}$ is an $N \times N$ matrix with entries i.i.d $\mathcal{N}\left(0, \sigma_{2}^{2}\right)$ and $\sigma_{2}^{2}=$ $\sigma_{N_{2}}^{2} \operatorname{Tr}\left(\mathbf{R}_{r}^{-1}\right) / P_{t}$.

Since we assume the channel reciprocity, using (3) and (4), we can write the source-to-relay channels $\mathbf{H}_{m i}$ as follows:

$$
\begin{aligned}
\mathbf{H}_{m i}= & \mathbf{R}_{r}^{\frac{1}{2}} \mathbf{H}_{i} \mathbf{R}_{i}^{\frac{1}{2}}=\mathbf{R}_{r}^{\frac{1}{2}} \overline{\mathbf{H}}_{i} \mathbf{R}_{i}^{\frac{1}{2}}+\mathbf{R}_{r}^{\frac{1}{2}} \mathbf{E}_{i}^{T} \\
& \times\left[\mathbf{I}_{N}+\sigma_{i}^{2} \mathbf{R}_{i}^{-1}\right]^{-\frac{T}{2}} \mathbf{R}_{i}^{-\frac{T}{2}} \mathbf{R}_{i}^{\frac{1}{2}} \quad i=1,2 .
\end{aligned}
$$

Correlation matrices are symmetric, and for simplicity, we denote $\overline{\mathbf{H}}_{m i}=\mathbf{R}_{r}^{\frac{1}{2}} \overline{\mathbf{H}}_{i} \mathbf{R}_{i}^{\frac{1}{2}}$ and $\mathbf{E}_{m i}=$ $\mathbf{R}_{r}^{\frac{1}{2}} \mathbf{E}_{i}^{T}\left[\mathbf{I}_{N}+\sigma_{i}^{2} \mathbf{R}_{i}^{-1}\right]^{-\frac{T}{2}}$, where these represent each channel matrix with a mean part and an estimation error part. We use these estimated channels to design precoders and decoder.

\subsection{Physical layer network coding}

During data transmission, modulated symbol vectors are fed into sources 1 and 2, with each given as $\mathbf{x}_{1}=\left(x_{11}, x_{12}, x_{13}, \ldots, x_{1 N}\right)^{T}$ and $\mathbf{x}_{2}=\left(x_{21}, x_{22}, x_{23}, \ldots, x_{2 N}\right)^{T}$, where $x_{i i} \in \mathbb{C}$ and $\mathcal{E}_{\mathbf{x}}\left\{\mathbf{x}_{i} \mathbf{x}_{i}^{H}\right\}=\mathbf{I}_{N}(i=1,2)$. The relay node estimates the summation of modulated signals $\left(\mathbf{x}_{1}+\mathbf{x}_{2}\right)$ and transmits it during the next time slot. This is more general and a valid PNC scheme for any modulation alphabet. For simple modulation schemes like QPSK, this summation of two signals can be mapped to XOR of two transmitted unmodulated information [8]. During the next time slot, the modulated symbol of XOR version will then be transmitted. In summary, we can carry out PNC for any case if we design the precoders and decoders to minimize the MSE between received signal and summation of modulated signals.

During the first time slot, the received signal vector $\mathbf{y} \in$ $\mathbb{C}^{N \times 1}$ at the relay is given by the following:

$$
\mathbf{y}=\mathbf{G H}_{m 1} \mathbf{F}_{1} \mathbf{x}_{1}+\mathbf{G H}_{m 2} \mathbf{F}_{2} \mathbf{x}_{2}+\mathbf{G n},
$$

where $\mathbf{n} \sim \mathcal{C N}\left(0, \sigma^{2} \mathbf{I}_{N}\right)$. The relay node estimates the $\mathbf{x}_{1}+\mathbf{x}_{2}$, and this leads us to consider $N$ number of separate spatial streams. Therefore, the received signal at $i$ th stream $y_{i}$ can be used to obtain an estimate corresponding to $x_{1 i}+x_{2 i}$. This scheme reduces the complexity of the PNC mapping at the relay [32]. We denote the estimation of $x_{1 i}+x_{2 i}$ as $x_{3 i}$, and $x_{3 i}$ broadcasts to other nodes during the next time slot.

During the second time slot, the received signal vector $\mathrm{y}_{1} \in \mathbb{C}^{N \times 1}$ at the source 1 is given as follows:

$$
\mathbf{y}_{1}=\mathbf{G}_{1} \mathbf{H}_{b 1} \mathbf{F}_{r} \mathbf{x}_{3}+\mathbf{G}_{1} \mathbf{n}_{1},
$$

where $\mathbf{x}_{3}=\left(x_{31}, x_{32}, x_{33}, \ldots, x_{3 N}\right)^{T}$ and $\mathbf{n}_{1} \sim$ $\mathcal{C N}\left(0, \sigma^{2} \mathbf{I}_{N}\right)$. Similarly, received signal vector $\mathbf{y}_{2} \in \mathbb{C}^{N \times 1}$ at the source 2 is given as follows:

$$
\mathbf{y}_{2}=\mathbf{G}_{2} \mathbf{H}_{b 1} \mathbf{F}_{r} \mathbf{x}_{3}+\mathbf{G}_{2} \mathbf{n}_{1},
$$

where $\mathbf{n}_{2} \sim \mathcal{C N}\left(0, \sigma^{2} \mathbf{I}_{N}\right)$. A source node estimates $\mathbf{x}_{3}$ and filter out its transmitted symbol. This gives the desired symbol, which is transmitted by the other source node. Here, the PNC operation can be considered independently at each relay antenna. In the case of nodes with different number of antennas, the maximum number of independent flows is limited to the minimum number of antennas at all nodes. The PNC operation is then considered in 
a similar manner as for the case where there are equal number of antennas at the nodes.

Accuracy of the PNC mapping is dependent on the estimated summation of two symbols. Therefore, it is evident that the optimum joint design is required to have accurate estimation process. Problem formulation and the solving method for designing optimum precoders and decoders are described in the next sections of the paper.

\subsection{Problem formulation}

As two-way communications have two phases, we can consider the analysis separately for both phases. For each phase, the total power can be limited, which can occur in many practical scenarios. Therefore, we consider $P_{T}$ as the maximum total transmitted power available in each time slot. A similar problem formulation and solving procedure is valid for individual power constraints of nodes.

\subsubsection{Multiple-access stage}

In this stage, both source nodes transmit to relay, and the transmitted powers of source nodes should satisfy the following constraint:

$$
\operatorname{Tr}\left(\mathbf{F}_{1} \mathbf{F}_{1}^{H}\right)+\operatorname{Tr}\left(\mathbf{F}_{2} \mathbf{F}_{2}^{H}\right) \leq P_{T}
$$

The received signal (6) during the MA stage is used to estimate $\mathbf{x}_{1}+\mathbf{x}_{2}$. The estimation error vector $\mathbf{e}_{m}$ can be defined as follows:

$$
\mathbf{e}_{m}=\mathbf{G H}_{m 1} \mathbf{F}_{1} \mathbf{x}_{1}+\mathbf{G H}_{m 2} \mathbf{F}_{2} \mathbf{x}_{2}+\mathbf{G n}-\mathbf{x}_{1}-\mathbf{x}_{2}
$$

Data streams may need different quality of service (QoS). We facilitate this by introducing weights for different streams. A diagonal $N \times N$ positive definite weight matrix $\mathbf{W}$ is used for that purpose. We express WMSE at the MA stage as follows:

$$
\begin{aligned}
\operatorname{WMSE}_{m} & =\mathcal{E}_{\mathbf{x}, \mathbf{n}}\left\{\left\|\mathbf{W}^{1 / 2} \mathbf{e}_{m}\right\|^{2}\right\} \\
& =\mathcal{E}_{\mathbf{x}, \mathbf{n}}\left\{\operatorname{Tr}\left(\mathbf{W}^{1 / 2} \mathbf{e}_{m} \mathbf{e}_{m}^{H} \mathbf{W}^{H / 2}\right)\right\} \\
& =\operatorname{Tr}\left(\mathbf{W} \mathcal{E}_{\mathbf{x}, \mathbf{n}}\left\{\mathbf{e}_{m} \mathbf{e}_{m}^{H}\right\}\right),
\end{aligned}
$$

where $\mathcal{E}_{\mathbf{x}, \mathbf{n}}\left\{\mathbf{e}_{m} \mathbf{e}_{m}^{H}\right\}$ is given by the following:

$$
\begin{aligned}
& \mathcal{E}_{\mathbf{x}, \mathbf{n}}\left\{\mathbf{e}_{m} \mathbf{e}_{m}^{H}\right\}=\left(\mathbf{G H}_{m 1} \mathbf{F}_{1}-\mathbf{I}_{N}\right)\left(\mathbf{G H}_{m 1} \mathbf{F}_{1}-\mathbf{I}_{N}\right)^{H} \\
& +\left(\mathbf{G H}_{m 2} \mathbf{F}_{2}-\mathbf{I}_{N}\right)\left(\mathbf{G H}_{m 2} \mathbf{F}_{2}-\mathbf{I}_{N}\right)^{H}+\sigma^{2} \mathbf{G G}^{H} .
\end{aligned}
$$

We use $\mathcal{E}_{\mathbf{x}, \mathbf{n}}\left\{\mathbf{x}_{1} \mathbf{x}_{1}^{H}\right\}=\mathbf{I}_{N}, \mathcal{E}_{\mathbf{x}, \mathbf{n}}\left\{\mathbf{x}_{2} \mathbf{x}_{2}^{H}\right\}=\mathbf{I}_{N}$, $\mathcal{E}_{\mathbf{x}, \mathbf{n}}\left\{\mathbf{x}_{1} \mathbf{x}_{2}^{H}\right\}=\mathbf{0}_{N \times N}, \mathcal{E}_{\mathbf{x}, \mathbf{n}}\left\{\mathbf{x}_{1} \mathbf{n}^{H}\right\}=\mathbf{0}_{N \times N}, \mathcal{E}_{\mathbf{x}, \mathbf{n}}\left\{\mathbf{x}_{2} \mathbf{n}^{H}\right\}=$ $\mathbf{0}_{N \times N}$ and $\mathcal{E}_{\mathbf{x}, \mathbf{n}}\left\{\mathbf{n n}^{H}\right\}=\sigma^{2} \mathbf{I}_{N}$ to obtain (12).

We need to minimize $\mathrm{WMSE}_{m}$ during the MA stage subject to the total power constraint to find optimum precoders and decoders. However, for given channel instances of $\mathbf{H}_{m 1}$ and $\mathbf{H}_{m 2}$, the estimation error becomes a random variable. We have to consider this channel estimation error with $\mathrm{WMSE}_{m}$. The error has a Gaussian distribution, and we focus on the expected value of $\mathrm{WMSE}_{m}$, given as follows:

$$
\begin{aligned}
\mathcal{E}_{\mathbf{E}}\left\{\mathrm{WMSE}_{m}\right\}= & \mathcal{E}_{\mathbf{E}}\left\{\operatorname { T r } \left(\mathbf{W}\left(\mathbf{G H}_{m 1} \mathbf{F}_{1}-\mathbf{I}_{N}\right)\right.\right. \\
& \times\left(\mathbf{G H} \mathbf{H}_{m 1} \mathbf{F}_{1}-\mathbf{I}_{N}\right)^{H}+\mathbf{W}\left(\mathbf{G H}_{m 2} \mathbf{F}_{2}-\mathbf{I}_{N}\right) \\
& \left.\left.\times\left(\mathbf{G H}_{m 2} \mathbf{F}_{2}-\mathbf{I}_{N}\right)^{H}+\sigma^{2} \mathbf{W G G}^{H}\right)\right\} .
\end{aligned}
$$

Channel estimates in (5) consist of the MMSE value and the error part as $\mathbf{H}_{m i}=\overline{\mathbf{H}}_{m i}+\mathbf{E}_{m i}$ for $i=1,2$. Therefore, expanding (13) into the following:

$$
\begin{aligned}
\mathcal{E}_{\mathbf{E}}\left\{\mathrm{WMSE}_{m}\right\}= & \sum_{i=1}^{2} \mathcal{E}_{\mathbf{E}}\left\{\operatorname{Tr}\left(\mathbf{W G} \overline{\mathbf{H}}_{m i} \mathbf{F}_{i} \mathbf{F}_{i}^{H} \overline{\mathbf{H}}_{m i}^{H} \mathbf{G}^{H}\right)\right\} \\
& +\mathcal{E}_{\mathbf{E}}\left\{\operatorname{Tr}\left(\mathbf{W} \mathbf{G} \mathbf{E}_{m i} \mathbf{F}_{i} \mathbf{F}_{i}^{H} \overline{\mathbf{H}}_{m i}^{H} \mathbf{G}^{H}\right)\right\} \\
& +\sum_{i=1}^{2} \mathcal{E}_{\mathbf{E}}\left\{\operatorname{Tr}\left(\mathbf{W G} \overline{\mathbf{H}}_{m i} \mathbf{F}_{i} \mathbf{F}_{i}^{H} \mathbf{E}_{m i}^{H} \mathbf{G}^{H}\right)\right\} \\
& +\mathcal{E}_{\mathbf{E}}\left\{\operatorname{Tr}\left(\mathbf{W G} \mathbf{E}_{m i} \mathbf{F}_{i} \mathbf{F}_{i}^{H} \mathbf{E}_{m i}^{H} \mathbf{G}^{H}\right)\right\} \\
& -\sum_{i=1}^{2} \mathcal{E}_{\mathbf{E}}\left\{\operatorname { T r } \left(\mathbf{W} \mathbf{G} \overline{\mathbf{H}}_{m i} \mathbf{F}_{i}-\mathbf{W} \mathbf{F}_{i}^{H} \overline{\mathbf{H}}_{m i}^{H} \mathbf{G}^{H}\right.\right. \\
& \left.\left.+\operatorname{WG} \mathbf{E}_{m i} \mathbf{F}_{i}-\mathbf{W} \mathbf{F}_{i}^{H} \mathbf{E}_{m i}^{H} \mathbf{G}^{H}\right)\right\} \\
& +\operatorname{Tr}\left(2 \mathbf{W}+\sigma^{2} \mathbf{W G} \mathbf{G}^{H}\right)
\end{aligned}
$$

since $\mathcal{E}_{\mathbf{E}}\left\{\mathbf{E}_{m i}\right\}=\mathbf{0}_{N \times N}$ (14) reduces to the following:

$$
\begin{aligned}
\mathcal{E}_{\mathbf{E}}\left\{\mathrm{WMSE}_{m}\right\}= & \sum_{i=1}^{2} \operatorname{Tr}\left(\mathbf{W G} \overline{\mathbf{H}}_{m i} \mathbf{F}_{i} \mathbf{F}_{i}^{H} \overline{\mathbf{H}}_{m i}^{H} \mathbf{G}^{H}\right) \\
& +\mathcal{E}_{\mathbf{E}}\left\{\operatorname{Tr}\left(\mathbf{W} \mathbf{G} \mathbf{E}_{m i} \mathbf{F}_{i} \mathbf{F}_{i}^{H} \mathbf{E}_{m i}^{H} \mathbf{G}^{H}\right)\right\} \\
& -\sum_{i=1}^{2} \operatorname{Tr}\left(\mathbf{W} \mathbf{G} \overline{\mathbf{H}}_{m i} \mathbf{F}_{i}-\mathbf{W} \mathbf{F}_{i}^{H} \overline{\mathbf{H}}_{m i}^{H} \mathbf{G}^{H}\right) \\
& +\operatorname{Tr}\left(2 \mathbf{W}+\sigma^{2} \mathbf{W} \mathbf{G G}^{H}\right)
\end{aligned}
$$

Moreover, we can expand the following term with the expectation as follows:

$$
\begin{aligned}
\mathcal{E}_{\mathbf{E}}\{ & \left.\operatorname{Tr}\left(\mathbf{W G} \mathbf{E}_{m i} \mathbf{F}_{i} \mathbf{F}_{i}^{H} \mathbf{E}_{m i}^{H} \mathbf{G}^{H}\right)\right\} \\
= & \mathcal{E}_{\mathbf{E}}\left\{\operatorname { T r } \left(\mathbf{W} \mathbf{G} \mathbf{R}_{r}^{\frac{1}{2}} \mathbf{E}_{i}^{T}\left[\mathbf{I}_{N}+\sigma_{i}^{2} \mathbf{R}_{i}^{-1}\right]^{-\frac{T}{2}}\right.\right. \\
& \left.\left.\times \mathbf{F}_{i} \mathbf{F}_{i}^{H}\left[\mathbf{I}_{N}+\sigma_{i}^{2} \mathbf{R}_{i}^{-1}\right]^{-\frac{T}{2}} \mathbf{E}_{i}^{*} \mathbf{R}_{r}^{\frac{1}{2}} \mathbf{G}^{H}\right)\right\} \\
= & \mathcal{E}_{\mathbf{E}}\left\{\operatorname { T r } \left(\mathbf{R}_{r}^{\frac{1}{2}} \mathbf{G}^{H} \mathbf{W} \mathbf{G} \mathbf{R}_{r}^{\frac{1}{2}} \mathbf{E}_{i}^{T}\left[\mathbf{I}_{N}+\sigma_{i}^{2} \mathbf{R}_{i}^{-1}\right]^{-\frac{T}{2}}\right.\right. \\
& \left.\left.\times \mathbf{F}_{i} \mathbf{F}_{i}^{H}\left[\mathbf{I}_{N}+\sigma_{i}^{2} \mathbf{R}_{i}^{-1}\right]^{-\frac{T}{2}} \mathbf{E}_{i}^{*}\right)\right\} \\
= & \mathcal{E}_{\mathbf{E}}\left\{\operatorname{Tr}\left(\mathbf{P}_{i} \mathbf{E}_{i}^{T} \mathbf{Q}_{i} \mathbf{E}_{i}^{*}\right)\right\},
\end{aligned}
$$

where we use $\mathbf{Q}_{i}=\left(\mathbf{I}_{N}+\sigma_{i}^{2} \mathbf{R}_{i}^{-1}\right)^{-\frac{1}{2}} \mathbf{F}_{i} \mathbf{F}_{i}^{H}\left(\mathbf{I}_{N}+\sigma_{i}^{2} \mathbf{R}_{i}^{-1}\right)^{-\frac{1}{2}}$ and $\mathbf{P}_{i}=\mathbf{R}_{r}^{\frac{1}{2}} \mathbf{G}^{H} \mathbf{W} \mathbf{G R}_{r}^{\frac{1}{2}}$. Next, we use relationships 
between trace and vectors to simplify (16) into the following:

$$
\begin{aligned}
\mathcal{E}_{\mathbf{E}}\left\{\operatorname{Tr}\left(\mathbf{P}_{i} \mathbf{E}_{i}^{T} \mathbf{Q}_{i} \mathbf{E}_{i}^{*}\right)\right\} & =\mathcal{E}_{\mathbf{E}}\left\{\operatorname{vec}\left(\mathbf{E}_{i}^{*}\right) \operatorname{vec}\left(\mathbf{P}_{i} \mathbf{E}_{i}^{T} \mathbf{Q}_{i}\right)\right\} \\
& =\mathcal{E}_{\mathbf{E}}\left\{\operatorname{vec}\left(\mathbf{E}_{i}^{*}\right)\left(\mathbf{Q}_{i} \otimes \mathbf{P}_{i}\right) \operatorname{vec}\left(\mathbf{E}_{i}^{T}\right)\right\} \\
& =\operatorname{Tr}\left(\left(\mathbf{Q}_{i} \otimes \mathbf{P}_{i}\right) \mathcal{E}\left\{\operatorname{vec}\left(\mathbf{E}_{i}^{T}\right) \operatorname{vec}\left(\mathbf{E}_{i}^{*}\right)\right\}\right) .
\end{aligned}
$$

We know that $\mathcal{E}\left\{\operatorname{vec}\left(\mathbf{E}_{i}^{T}\right) \operatorname{vec}\left(\mathbf{E}_{i}^{*}\right)\right\}=\sigma_{i}^{2} \mathbf{I}$ and using the relation $\operatorname{Tr}\left(\left(\mathbf{Q}_{i} \otimes \mathbf{P}_{i}\right)\right)=\operatorname{Tr}\left(\mathbf{Q}_{i}\right) \operatorname{Tr}\left(\mathbf{P}_{i}\right)$, we can find the following expression for (15):

$$
\begin{aligned}
\mathcal{E}_{\mathbf{E}}\left\{\mathrm{WMSE}_{m}\right\}=\sum_{i=1}^{2} \operatorname{Tr}\left(\mathbf{W} \mathbf{G} \overline{\mathbf{H}}_{m i} \mathbf{F}_{i} \mathbf{F}_{i}^{H} \overline{\mathbf{H}}_{m i}^{H} \mathbf{G}^{H}-\mathbf{W G} \overline{\mathbf{H}}_{m i} \mathbf{F}_{i}\right. \\
\left.\quad-\mathbf{W} \mathbf{F}_{i}^{H} \overline{\mathbf{H}}_{m i}^{H} \mathbf{G}^{H}+\mathbf{W}\right) \\
+\sum_{i=1}^{2} \sigma_{i}^{2} \operatorname{Tr}\left(\mathbf{Q}_{i}\right) \operatorname{Tr}\left(\mathbf{P}_{i}\right)+\operatorname{Tr}\left(\sigma^{2} \mathbf{W} \mathbf{G} \mathbf{G}^{H}\right)
\end{aligned}
$$

Next, we formulate the optimization problem to minimize expected value of $\mathrm{WMSE}_{m}$ under a limited available transmit power at source nodes as presented in Problem 1:

\section{Problem 1.}

$$
\begin{aligned}
\min _{\mathbf{F}_{1}, \mathbf{F}_{2}, \mathbf{G}} & \mathcal{E}_{\mathbf{E}}\left\{\mathrm{WMSE}_{m}\right\} \\
\text { subject to } & \operatorname{Tr}\left(\mathbf{F}_{1} \mathbf{F}_{1}^{H}\right)+\operatorname{Tr}\left(\mathbf{F}_{2} \mathbf{F}_{2}^{H}\right) \leq P_{T}
\end{aligned}
$$

This is a non-convex optimization problem. In Section 3, we propose an algorithm to solve this optimally.

\subsubsection{Broadcasting stage}

Estimated $\mathbf{x}_{1}+\mathbf{x}_{2}$, i.e., $\mathbf{x}_{3}$ broadcasts during this time slot. Relay uses $\mathbf{F}_{r}$ precoder and transmits $\mathbf{x}_{3}$ to both source nodes. Sources 1 and 2 now have $\mathbf{G}_{1}$ and $\mathbf{G}_{2}$ decoders, respectively. At source $i(=1,2)$, it estimates $\mathbf{x}_{3}$ and uses that to find desired symbol.

Similar to the MA stage, the joint design is considered to minimize the WMSE of received signals. We considered all nodes to satisfy a total power constraint for their transmission. Therefore, during the $\mathrm{BC}$ stage, transmit power at the relay node should satisfy the following constraint:

$$
\operatorname{Tr}\left(\mathbf{F}_{r} \mathbf{F}_{r}^{H}\right) \leq P_{T} / 2
$$

This constraint becomes $P_{T} / 2$ because $\mathcal{E}_{\mathbf{x}}\left\{\mathbf{x}_{3} \mathbf{x}_{3}^{H}\right\}$ is now equal to $2 \mathbf{I}_{N} ; \mathbf{e}_{b i}$ is the estimation error vector at $i$ th source. It is given as follows:

$$
\mathbf{e}_{b i}=\mathbf{G}_{i} \mathbf{H}_{b i} \mathbf{F}_{r} \mathbf{x}_{3}+\mathbf{G}_{i} \mathbf{n}_{i}-\mathbf{x}_{3} .
$$

We use the similar weights for different streams as in multiple-access analysis. WMSE at source $i$ is denoted as $\mathrm{WMSE}_{i}$ and is given by the following:

$$
\begin{aligned}
\mathrm{WMSE}_{i}= & 2 \mathbf{W}\left(\mathbf{G}_{i} \mathbf{H}_{b i} \mathbf{F}_{r}-\mathbf{I}_{N}\right)\left(\mathbf{G}_{i} \mathbf{H}_{b i} \mathbf{F}_{r}-\mathbf{I}_{N}\right)^{H} \\
& +\sigma^{2} \mathbf{W} \mathbf{G}_{i} \mathbf{G}_{i}^{H} \quad i=1,2 .
\end{aligned}
$$

$\mathbf{H}_{b i}$ has an error component. Therefore, the expected value of $\mathrm{WMSE}_{i}$ is considered in the optimum precoderdecoder design. A similar procedure as in the MA stage is valid to find the expected value of $\mathrm{WMSE}_{i}$. We find $\mathrm{WMSE}_{i}$ as follows:

$$
\begin{aligned}
& \begin{aligned}
\mathcal{E}_{\mathbf{E}}\left\{\mathbf{W M S E} \mathrm{W}_{i}\right\}= & 2 \operatorname{Tr}\left(\mathbf{W} \mathbf{G}_{i} \overline{\mathbf{H}}_{m i}^{T} \mathbf{F}_{r} \mathbf{F}_{r}^{H} \overline{\mathbf{H}}_{m i}^{*} \mathbf{G}_{i}^{H}\right. \\
& \left.\quad-\mathbf{W} \mathbf{G}_{i} \overline{\mathbf{H}}_{m i}^{T} \mathbf{F}_{r}-\mathbf{W} \mathbf{F}_{r}^{H} \overline{\mathbf{H}}_{m i}^{*} \mathbf{G}_{i}^{H}+\mathbf{W}\right) \\
& +2 \sigma_{i}^{2} \operatorname{Tr}\left(\mathbf{L}_{i}\right) \operatorname{Tr}(\mathbf{K}) \\
& +\operatorname{Tr}\left(\sigma^{2} \mathbf{W} \mathbf{G}_{i} \mathbf{G}_{i}^{H}\right) \quad i=1,2,
\end{aligned} \\
& \text { where } \mathbf{L}_{i}=\left(\mathbf{I}_{N}+\sigma_{i}^{2} \mathbf{R}_{i}^{-1}\right)^{-\frac{1}{2}} \mathbf{G}_{i}^{H} \mathbf{W} \mathbf{G}_{i}^{H}\left(\mathbf{I}_{N}+\sigma_{i}^{2} \mathbf{R}_{i}^{-1}\right)^{-\frac{1}{2}} \\
& \text { and } \mathbf{K}=\mathbf{R}_{r}^{\frac{1}{2}} \mathbf{F}_{r} \mathbf{F}_{r}^{H} \mathbf{R}_{r}^{\frac{1}{2}} .
\end{aligned}
$$

Both source nodes are trying to minimize expected values of the WMSE 1 and $\mathrm{WMSE}_{2}$ during the $\mathrm{BC}$ stage. We are not considering a greedy approach, i.e., every node is trying to minimize its own WMSE component. Equal proportions of WMSE are considered to find optimum precoder and decoders. Therefore, we consider the sum of two components, and the problem is formulated in Problem 2.

Problem 2.

$$
\begin{aligned}
\min _{\mathbf{F}_{r}, \mathbf{G}_{1}, \mathbf{G}_{2}} & \frac{1}{2} \mathcal{E}_{\mathbf{E}}\left\{\mathrm{WMSE}_{1}\right\}+\frac{1}{2} \mathcal{E}_{\mathbf{E}}\left\{\mathrm{WMSE}_{2}\right\} \\
\text { subject to } & \operatorname{Tr}\left(\mathbf{F}_{r} \mathbf{F}_{r}^{H}\right) \leq P_{T} / 2 .
\end{aligned}
$$

This problem is a non-convex problem, and we propose solutions in the next section.

\section{Optimum joint designs}

Here, we propose optimum algorithms to solve the nonconvex optimization Problems 1 and 2. We can prove that both problems have global minimums (Appendix), and we can achieve those in our proposed algorithms.

\subsection{Optimum precoder-decoder design for MA stage}

Here, we propose an algorithm to solve Problem 1 by dividing it into two sub-problems. Mainly, we can find two different sets of variables in this problem. Precoders can be categorized into one set of variables and the decoder into the other. With these observations, we proceed with the following method.

First, we consider precoders $\mathbf{F}_{1}$ and $\mathbf{F}_{2}$ to be fixed. We find $\mathbf{G}$ to minimize expected value of $\mathrm{WMSE}_{m}$. Next, we consider $\mathbf{G}$ to be fixed and $\mathbf{F}_{1}$ and $\mathbf{F}_{2}$ to be variables. 
These are identified as the two sub-problems of the original problem. The solutions of these two sub-problems are used in the next iteration. The problem is solved iteratively until $\mathbf{G}, \mathbf{F}_{1}$, and $\mathbf{F}_{2}$ converge to become fixed matrices.

\subsubsection{Sub-problem $1 A$}

We consider $\mathbf{F}_{1}$ and $\mathbf{F}_{2}$ as fixed. Therefore, the problem is reduced to the following form:

$$
\min _{\mathbf{G}} \mathcal{E}_{\mathbf{E}}\left\{\mathrm{WMSE}_{m}\right\}
$$

The power constraint is independent of $\mathbf{G}$. Therefore, we take derivative of the objective function and make that equal to zero. The solution for $\mathbf{G}$ is the optimum one during that iteration and is given as a function of $\mathbf{F}_{1}, \mathbf{F}_{2}$, and other parameters:

$$
\begin{aligned}
& \frac{\partial \mathcal{E}_{\mathbf{E}}\left\{\mathrm{WMSE}_{m}\right\}}{\partial \mathbf{G}^{*}}=0, \\
& \mathbf{G}=\left(\mathbf{F}_{1}^{H} \overline{\mathbf{H}}_{m 1}^{H}+\mathbf{F}_{2}^{H} \overline{\mathbf{H}}_{m 2}^{H}\right) \\
& \times\left(\overline{\mathbf{H}}_{m 1} \mathbf{F}_{1} \mathbf{F}_{1}^{H} \overline{\mathbf{H}}_{m 1}^{H}+\overline{\mathbf{H}}_{m 2} \mathbf{F}_{2} \mathbf{F}_{2}^{H} \overline{\mathbf{H}}_{m 2}^{H}\right. \\
& \left.+\sigma_{1}^{2} \operatorname{Tr}\left(\mathbf{Q}_{1}\right) \mathbf{R}_{r}+\sigma_{2}^{2} \operatorname{Tr}\left(\mathbf{Q}_{2}\right) \mathbf{R}_{r}+\sigma^{2} \mathbf{I}_{N}\right)^{-1} \text {. }
\end{aligned}
$$

We considered complex-valued matrix function differentiation as in [33] to obtain the results.

\subsubsection{Sub-problem $1 B$}

We consider a similar problem as in (19) and keep G as fixed. Therefore, we have two variables, $\mathbf{F}_{1}$ and $\mathbf{F}_{2}$. Optimization problem can be reformulated as follows:

$$
\begin{gathered}
\min _{\mathbf{F}_{1}, \mathbf{F}_{2}} \sum_{i=1}^{2} \operatorname{Tr}\left(\mathbf{W} \mathbf{G} \overline{\mathbf{H}}_{m i} \mathbf{F}_{i} \mathbf{F}_{i}^{H} \overline{\mathbf{H}}_{m i}^{H} \mathbf{G}^{H}-\mathbf{W} \mathbf{G} \overline{\mathbf{H}}_{m i} \mathbf{F}_{i}\right. \\
\left.-\mathbf{W} \mathbf{F}_{i}^{H} \overline{\mathbf{H}}_{m i}^{H} \mathbf{G}^{H}+\mathbf{W}\right) \\
+\sum_{i=1}^{2} \sigma_{i}^{2} \operatorname{Tr}\left(\mathbf{Q}_{i}\right) \operatorname{Tr}\left(\mathbf{P}_{i}\right)+\operatorname{Tr}\left(\sigma^{2} \mathbf{W} \mathbf{G} \mathbf{G}^{H}\right)
\end{gathered}
$$$$
\text { subject to } \operatorname{Tr}\left(\mathbf{F}_{1} \mathbf{F}_{1}^{H}\right)+\operatorname{Tr}\left(\mathbf{F}_{2} \mathbf{F}_{2}^{H}\right) \leq P_{T} \text {. }
$$

A variable transformation is considered before solving this problem. We define new matrix variables $\mathbf{F} \in \mathbb{C}^{2 N \times 2 N}$ as $\mathbf{F}=\left(\begin{array}{lll}\mathbf{F}_{1} & \mathbf{0}_{N \times N} ; \mathbf{0}_{N \times N} & \mathbf{F}_{2}\end{array}\right)$. We also define the following matrices $\mathbf{A}$ and $\mathbf{C}$ to simplify the other parameters, where $\mathbf{A}=\left(\mathbf{W}^{\frac{1}{2}} \mathbf{G} \overline{\mathbf{H}}_{m 1} \mathbf{0}_{N \times N} ; \mathbf{0}_{N \times N} \mathbf{W}^{\frac{1}{2}} \mathbf{G} \overline{\mathbf{H}}_{m 2}\right)$ and

$$
\mathbf{C}=\left(\begin{array}{cc}
\sqrt{\sigma_{1}^{2} \operatorname{Tr}\left(\mathbf{P}_{1}\right)}\left(\mathbf{I}_{N}+\sigma_{1}^{2} \mathbf{R}_{1}^{-1}\right)^{-\frac{1}{2}} & \mathbf{0}_{N \times N} \\
\mathbf{0}_{N \times N} & \sqrt{\sigma_{2}^{2} \operatorname{Tr}\left(\mathbf{P}_{2}\right)}\left(\mathbf{I}_{N}+\sigma_{2}^{2} \mathbf{R}_{2}^{-1}\right)^{-\frac{1}{2}}
\end{array}\right) .
$$

The reformulated optimization problem is then given as follows:

$$
\begin{array}{cl}
\min _{\mathbf{F}} & \operatorname{Tr}\left(\mathbf{F}^{H} \mathbf{A}^{H} \mathbf{A F}\right)+\operatorname{Tr}\left(\mathbf{F}^{H} \mathbf{C}^{H} \mathbf{C F}\right)-2 \Re\left(\operatorname{Tr}\left(\mathbf{W}^{\frac{1}{2}} \mathbf{A F}\right)\right) \\
& +\operatorname{Tr}\left(2 \mathbf{W}+\sigma^{2} \mathbf{W} \mathbf{G G}^{H}\right) \\
\text { s.t } & \operatorname{Tr}\left(\mathbf{F F}^{H}\right) \leq P_{T}
\end{array}
$$

where $\mathbf{A}^{H} \mathbf{A}$ is a positive semi-definite matrix. This is convex and is known as the quadrature matrix programming problem. We transpose this into a quadratically constrained quadratic programming (QCQP) problem which is given by the following:

$$
\begin{array}{cc}
\min _{\mathbf{z}} & \operatorname{vec}(\mathbf{F})^{*}\left(\mathbf{I}_{2 N} \otimes\left(\mathbf{A}^{H} \mathbf{A}\right)+\mathbf{I}_{2 N} \otimes\left(\mathbf{C}^{H} \mathbf{C}\right)\right) \operatorname{vec}(\mathbf{F}) \\
& -2 \Re\left(\operatorname{vec}\left(\left(\left(\mathbf{W}^{\frac{1}{2}} \otimes \mathbf{I}_{N}\right) \mathbf{A}\right)^{H}\right)^{*} \operatorname{vec}(\mathbf{F})\right)+c_{m} \\
\text { s.t } & \operatorname{vec}(\mathbf{F})^{*}\left(\mathbf{I}_{2 N} \otimes \mathbf{I}_{2 N}\right) \operatorname{vec}(\mathbf{F}) \leq P_{T},
\end{array}
$$

where $\mathbf{z}=\operatorname{vec}(\mathbf{F})$ and $c_{m}=\operatorname{Tr}\left(2 \mathbf{W}+\sigma^{2} \mathbf{W} \mathbf{G G}^{H}\right)$. This can be easily solved with QCQP solvers, which ultimately give optimum matrices of $\mathbf{F}_{1}$ and $\mathbf{F}_{2}$ in that iteration. In the numerical analysis, we used interior point method to solve this sub-problem.

We solve Problem 1 using the two sub-problems mentioned. First, we start by fixing $\mathbf{F}_{1}$ and $\mathbf{F}_{2}$, giving initial values. Next, we solve sub-problem $1 \mathrm{~A}$ to find the optimum $\mathbf{G}$. We then use $\mathbf{G}$ to solve sub-problem $1 \mathrm{~B}$, which gives $\mathbf{F}_{1}$ and $\mathbf{F}_{2}$. These $\mathbf{F}_{1}$ and $\mathbf{F}_{2}$ will be used again to solve the sub-problem $1 \mathrm{~A}$, which updates the optimum G. We solve iteratively until the problem gives convergent solutions. The final algorithm is given as follows:

The proposed algorithm converges rapidly with a small number of iterations. Initialization point does not have any effect on the final convergence point. We find that this always reaches the global optimum.

\subsection{Optimum precoder-decoder design for BC stage}

During the $\mathrm{BC}$ stage, the requirement is that source nodes should find decoders and the relay node should find the precoder. Those can be found by solving Problem 2 . Similar to the previous stage, we can use an iterative algorithm to find the optimum design. First, we consider the fixed $\mathbf{F}_{r}$ matrix, and find $\mathbf{G}_{1}$ and $\mathbf{G}_{2}$. Next, we fix $\mathbf{G}_{1}$ and $\mathbf{G}_{2}$ and find $\mathbf{F}_{r}$. These can be identified as two sub-problems of the original Problem 2.

\subsubsection{Sub-problem $2 A$}

We consider $\mathbf{F}_{r}$ as fixed, and Problem 2 reduces to the following:

$$
\min _{\mathbf{G}_{1}, \mathbf{G}_{2}} \frac{1}{2} \mathcal{E}_{\mathbf{E}}\left\{\mathrm{WMSE}_{1}\right\}+\frac{1}{2} \mathcal{E}_{\mathbf{E}}\left\{\mathrm{WMSE}_{2}\right\} .
$$




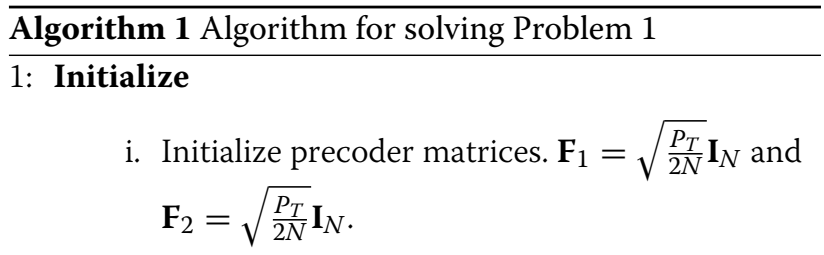

\section{2: Repeat}

i. Fix $\mathbf{F}_{1}$ and $\mathbf{F}_{2}$ obtained from (2.ii.) (Or (1.i) at initial point). Solve sub-problem $1 \mathrm{~A}$ to find optimum $\mathbf{G}$.

ii. Fix $\mathbf{G}$ obtained from (2.i). Solve sub-problem $1 \mathrm{~B}$ to find optimum $\mathbf{F}$. Then obtain $\mathbf{F}_{1}$ and $\mathbf{F}_{2}$.

\section{3: Until}

i. $\mathrm{WMSE}_{m}$ reduces during each step. Continue till it converges, i.e., $\left|\mathrm{WMSE}_{m}^{k+1}-\mathrm{WMSE}_{m}^{k}\right| \leq \epsilon$, where $k$ denotes the iteration number and $\epsilon(<<1)$ is a positive constant.

The power constraint is independent of $\mathbf{G}_{1}$ and $\mathbf{G}_{2}$. Therefore, we can derive the objective function with $\mathbf{G}_{1}$ and $\mathbf{G}_{2}$ and make those equal to zero. Solutions are given as follows:

$$
\begin{aligned}
\mathbf{G}_{i}^{\mathrm{opt}}= & \left(2 \mathbf{F}_{r}^{H} \overline{\mathbf{H}}_{m i}^{*}\right)\left(2 \overline{\mathbf{H}}_{m i}^{T} \mathbf{F}_{r} \mathbf{F}_{r}^{H} \overline{\mathbf{H}}_{m i}^{*}+2 \sigma_{i}^{2} \operatorname{Tr}(\mathbf{K})\right. \\
& \left.\times\left(\mathbf{I}_{N}+\sigma_{i}^{2} \mathbf{R}_{i}^{-1}\right)^{-1}+\sigma^{2} \mathbf{I}_{N}\right)^{-1} \quad i=1,2 .
\end{aligned}
$$

\subsubsection{Sub-problem $2 B$}

In here, we consider $\mathbf{G}_{1}$ and $\mathbf{G}_{2}$ as fixed. Therefore, we have one variable $\mathbf{F}_{r}$. The second sub-problem can be found as follows:

$$
\begin{aligned}
& \min _{\mathbf{F}_{r}} \sum_{i=1}^{2} \operatorname{Tr}\left(\mathbf{W} \mathbf{G}_{i} \overline{\mathbf{H}}_{m i}^{T} \mathbf{F}_{r} \mathbf{F}_{r}^{H} \overline{\mathbf{H}}_{m i}^{*} \mathbf{G}_{i}^{H}-\mathbf{W} \mathbf{G}_{i} \overline{\mathbf{H}}_{m i}^{T} \mathbf{F}_{r}\right.\left.-\mathbf{W} \mathbf{F}_{r}^{H} \overline{\mathbf{H}}_{m i}^{*} \mathbf{G}_{i}^{H}+\mathbf{W}\right) \\
&+\sum_{i=1}^{2} \sigma_{i}^{2} \operatorname{Tr}\left(\mathbf{L}_{i}\right) \operatorname{Tr}(\mathbf{K})+\frac{1}{2} \operatorname{Tr}\left(\sigma^{2} \mathbf{W} \mathbf{G}_{i} \mathbf{G}_{i}^{H}\right)
\end{aligned}
$$

subject to $\operatorname{Tr}\left(\mathbf{F}_{r} \mathbf{F}_{r}^{H}\right) \leq P_{T} / 2$

We can transform this problem into QCQP, which is given by the following:

$$
\begin{aligned}
\min _{\mathbf{z}} \frac{1}{2} \operatorname{vec}\left(\mathbf{F}_{r}\right)^{*}\left(\sum_{i=1}^{2} \mathbf{I}_{N} \otimes\left(\mathbf{A}_{i}^{H} \mathbf{A}_{i}\right)\right. \\
\left.+\sum_{i=1}^{2} \mathbf{I}_{N} \otimes\left(\mathbf{C}_{i}^{H} \mathbf{C}_{i}\right)\right) \operatorname{vec}\left(\mathbf{F}_{r}\right) \\
-\Re\left(\operatorname{vec}\left((\mathbf{B})^{H}\right)^{*} \operatorname{vec}\left(\mathbf{F}_{r}\right)\right)+c_{b}
\end{aligned}
$$

subjected to $\operatorname{vec}\left(\mathbf{F}_{r}\right)^{*}\left(\mathbf{I}_{N} \otimes \mathbf{I}_{N}\right) \operatorname{vec}\left(\mathbf{F}_{r}\right) \leq P_{T} / 2$,

where $\mathbf{z}=\operatorname{vec}\left(\mathbf{F}_{r}\right), \mathbf{A}_{i}=\mathbf{W}^{\frac{1}{2}} \mathbf{G}_{i} \overline{\mathbf{H}}_{m i}^{T}, \mathbf{C}_{i}=\sqrt{\sigma_{i}^{2} \operatorname{Tr}\left(\mathbf{L}_{i}\right)} \mathbf{R}_{r}^{\frac{1}{2}}$, $\mathbf{B}=\mathbf{W} \mathbf{G}_{1} \overline{\mathbf{H}}_{m 1}^{T}+\mathbf{W} \mathbf{G}_{2} \overline{\mathbf{H}}_{m 2}^{T}$, and $c_{b}=\operatorname{Tr}(2 \mathbf{W}+$ $\left.\frac{1}{2} \sigma^{2} \mathbf{W} \mathbf{G}_{1} \mathbf{G}_{1}^{H}+\frac{1}{2} \sigma^{2} \mathbf{W} \mathbf{G}_{2} \mathbf{G}_{2}^{H}\right)$. This is a convex problem and can be solved with any QCQP solver. We used interior point method to solve this in our numerical analysis. Finally, Problem 2 can be solved with the following algorithm.

\section{Algorithm 2 Algorithm for solving Problem 2 \\ 1: Initialize \\ i. Initialize precoder matrices. $\mathbf{F}_{r}=\sqrt{\frac{P_{T}}{4 N}} \mathbf{I}_{N}$.}

\section{2: Repeat}

i. Fix $\mathbf{F}_{r}$ obtained from (2.ii.) (Or (1.i) at initial point). Solve sub-problem $2 \mathrm{~A}$ to find optimum $\mathbf{G}_{1}$, and $\mathbf{G}_{2}$.

ii. Fix $\mathbf{G}_{1}$, and $\mathbf{G}_{2}$ obtained from (2.i). Solve sub-problem $2 \mathrm{~B}$ to find optimum $\mathbf{F}_{r}$.

\section{3: Until}

(a) $\mathrm{WMSE}_{1}+\mathrm{WMSE}_{2}$ reduces in each step. Continue till it converges, i.e., $\left|\mathrm{WMSE}_{1}^{k+1}+\mathrm{WMSE}_{2}^{k+1}-\mathrm{WMSE}_{1}^{k}-\mathrm{WMSE}_{2}^{k}\right| \leq$ $\epsilon$, where $k$ denotes the iteration number, and $\epsilon(<<1)$ is a positive constant.

The proposed algorithm converges with a small number of iterations, and initial point does not have any effect on the final solution. As we explain in the Appendix, this reaches global optimum.

We use these two algorithms to find precoders and decoders in both MA and BC stages. These precoder and decoder matrices are dependent on the instantaneous channel information, and nodes dynamically adjust according to the CSI.

\section{Numerical results}

We use proposed algorithms to design precoders and decoders and to identify effects of channel estimation error, correlation coefficients, relay location, and weight 
parameters on error probability. Rayleigh fading channels are considered, and relay location is normalized from the source 1-to-relay distance. Numerical simulations are also assume that the transmitted symbols be uniformly distributed with unit magnitude. During the MA stage, the relay receiver estimates the summation of symbols. During the $\mathrm{BC}$ stage, source nodes estimate the broadcast symbol by the relay node (estimated sum of two symbols). We have considered WMSE minimization problems at both MA and BC stages. Therefore, we focus on the error analysis after a complete cycle. Antenna correlation of source 1 is defined by $\left(\mathbf{R}_{1}\right)_{i j}=\rho_{S 1}^{|i-j|}$, where $\rho_{S 1}$ is the correlation coefficient. A similar definition is used for $\mathbf{R}_{2}$ and $\mathbf{R}_{r}$ with correlation coefficients $\rho_{S 2}$ and $\rho_{R}$, respectively.

Figure 2 shows the average BER (ABER) performance with the transmit signal-to-noise ratio (SNR) $(\gamma)$ of a modulated symbol. Performance metric ABER considers average error rates at both source nodes after a complete cycle. The weighting matrix is considered as $\mathbf{W}=\frac{1}{N} \mathbf{I}_{N}$, the relay is located at midpoint $(\mathrm{d}=1)$, the number of antennas is $N=2$, convergence constant $\epsilon=0.0001$, and the total transmit power $P_{T}$ is selected such that $P_{T} / \sigma^{2}=$ $8 \gamma$. We consider both perfect and imperfect channel estimation scenarios with different relay correlation coefficients $\rho_{R}$. Here, the estimation error part $\mathbf{E}_{m i}$ of channel estimation is considered as in (5), and the error variance $\sigma_{i}^{2}$ is used to quantify the contribution of the error. The error variance $\sigma_{i}^{2}$ is obtained with $\sigma_{i}^{2}=\sigma_{N_{i}}^{2} \operatorname{Tr}\left(\mathbf{R}_{r}^{-1}\right) / P_{t}$. In the simulations, we change $P_{t} / \sigma_{N_{i}}^{2}$ to vary the $\sigma_{i}^{2}$. As seen in the Figure 2, the joint precoder-decoder design with the perfect channel knowledge performs better than the rest. When the estimation error increases, the performance reduces. This figure also shows the relay antenna correlation effect on the average BER. The joint design for the perfect channels is highly sensitive to antenna correlation of the relay. When the estimation error becomes higher, the antenna correlation has less effect, which can be seen from the case having $\sigma_{i}^{2}=0.02 . P_{t} / \sigma_{N_{i}}^{2}$ is considered

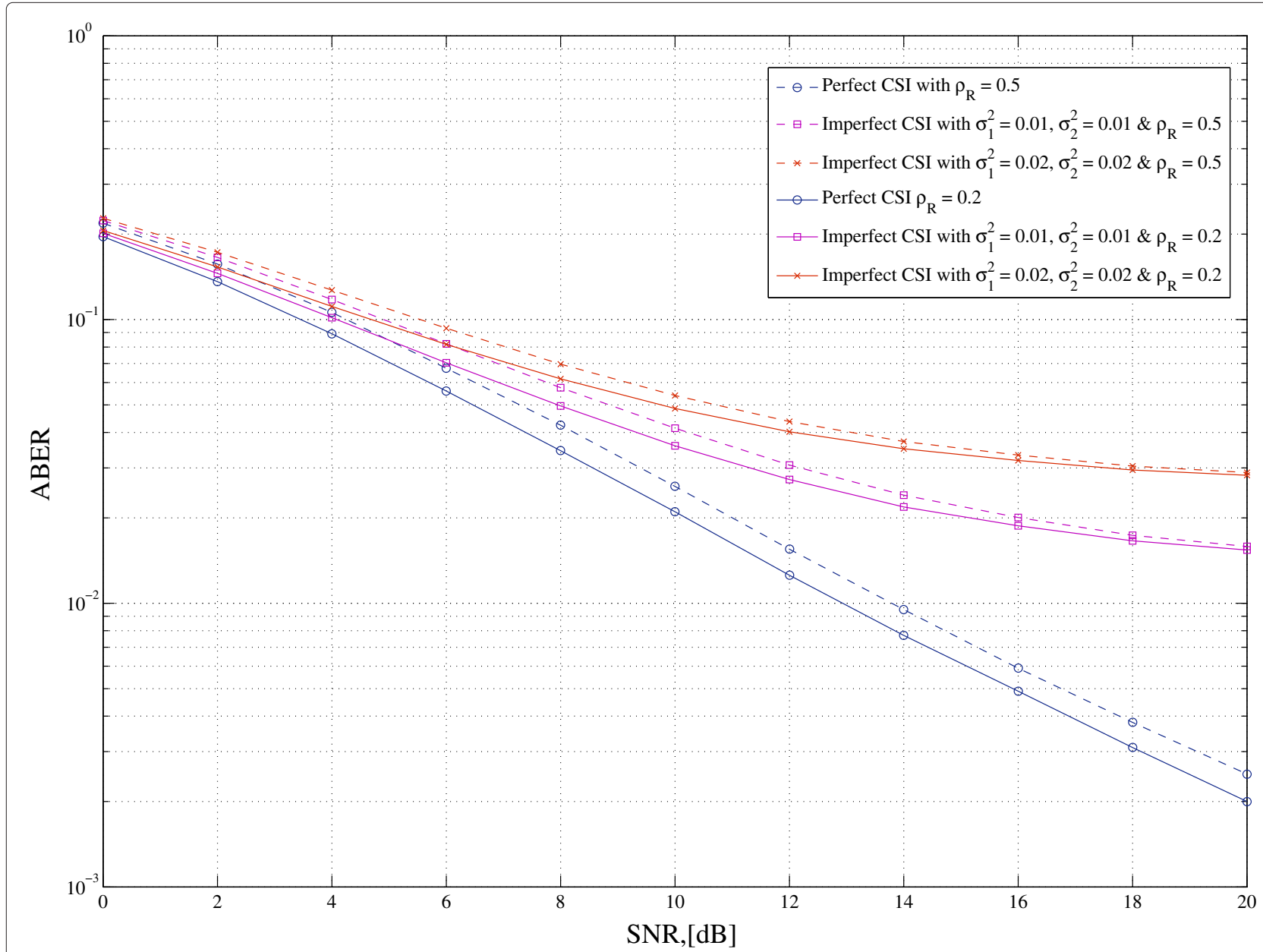

Figure 2 ABER versus transmit SNR: relay antenna correlation and estimation error. Average BER variation with SNR $(\gamma)$ for different relay antenna correlations and estimation error variances. $N=2, P_{T} / \sigma^{2}=8 \gamma$. 
as 21.2 and $20.2 \mathrm{~dB}$ for cases $\rho_{R}=0.5$ and $\rho_{R}=0.2$, respectively. Here, the difference in the error performance is very small. Similarly, Figure 3 presents the case when all nodes have antenna correlation. It is clear that when all nodes have some amount of correlation, the performances become degraded. A significant variance is visible with a small channel estimation error (or perfect estimation).

Next, we consider two other design schemes to compare the benefits of the proposed scheme. The first scheme considers signal processing at the source nodes during both MA and BC stages. During the MA stage, the optimum precoder design is considered to minimize WMSE at the source nodes. Here, the decoder is not taken into account. Similarly, at the BC stage, the decoder design is considered to minimize WMSE, where the precoder at the relay is not considered. The second scheme is the opposite of the first scheme. During the MA stage, the decoder design is considered at the relay node. During the $\mathrm{BC}$ stage, only the precoder design is considered. These two schemes are useful to find how beneficial the joint design is compared to other possible designs. Figure 4 shows the ABER variation of these three schemes with the transmit SNR. We consider $\mathbf{W}=\frac{1}{N} \mathbf{I}_{N}, N=2, \epsilon=$ $0.0001, P_{T} / \sigma^{2}=8 \gamma$, and $d=1$. It shows that the joint design scheme performs better than the rest. In the low SNR region, the first design scheme gives a small variation in ABER performance, whereas when SNR increases, the joint design has a significant performance improvement. This difference is reduced when the error variance becomes high.

In Figure 5, we considered the ABER variation with normalized distance $d$. We consider error rates of bidirectional transmissions and also their corresponding average. We consider $N=2, \epsilon=0.0001, \gamma=4 \mathrm{~dB}$, and $P_{T} / \sigma^{2}=$ $32 \mathrm{~dB}$. It can be seen that the joint design performs better than the rest for all relay locations. However, when the relay is near one source, the precoder design also gives better results.

We consider different weight parameters to observe their effect on MSE. Figure 6 shows the average MSE (AMSE) of the received signal at the first antenna of the relay (during MA stage) with the transmit

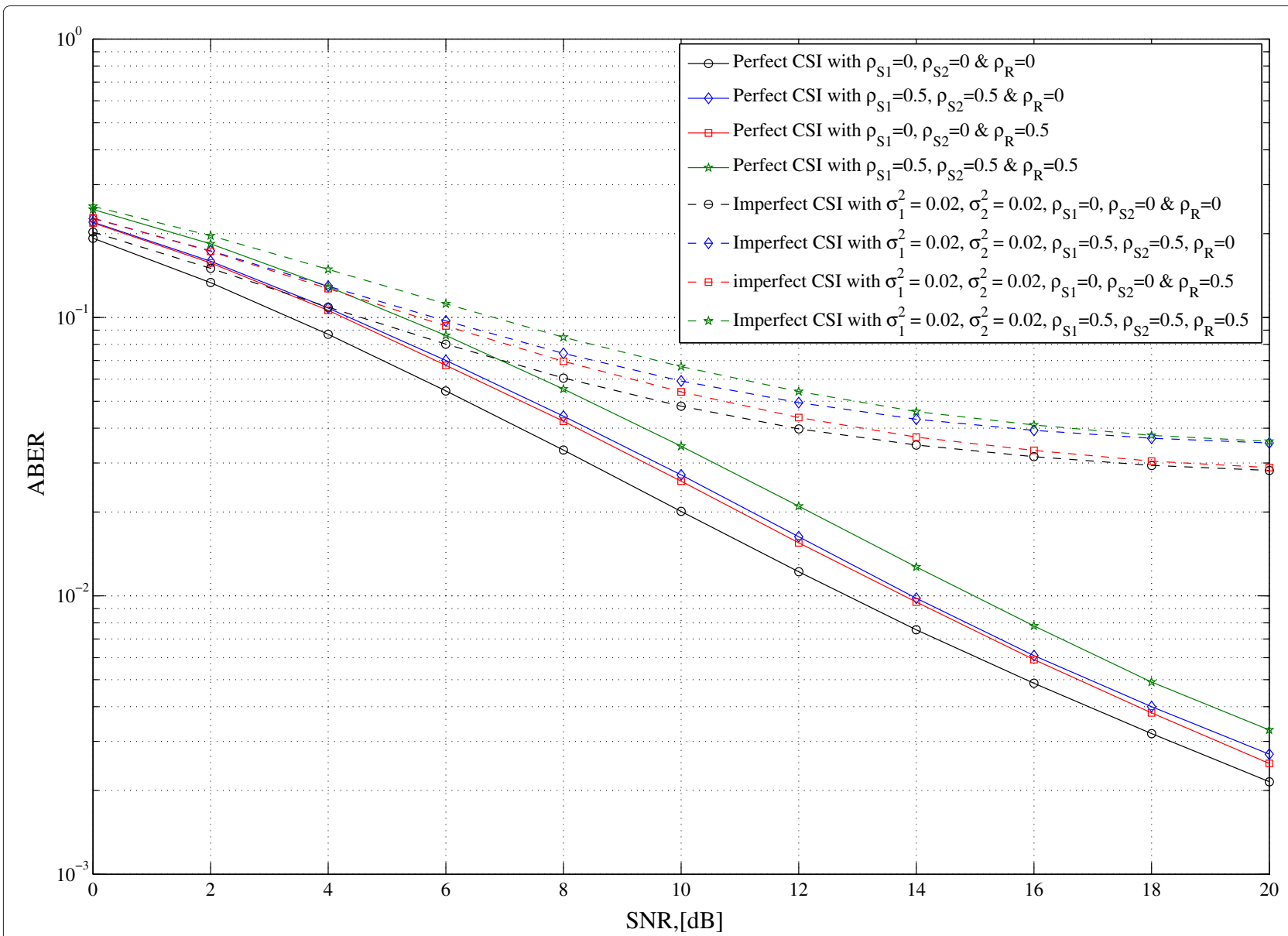

Figure 3 ABER versus transmit SNR: both relay and source antenna correlations and estimation error. Average BER variation with SNR $(\gamma)$ for different relay and source node antenna correlations and estimation error variances. $N=2, P_{T} / \sigma^{2}=8 \gamma$. 


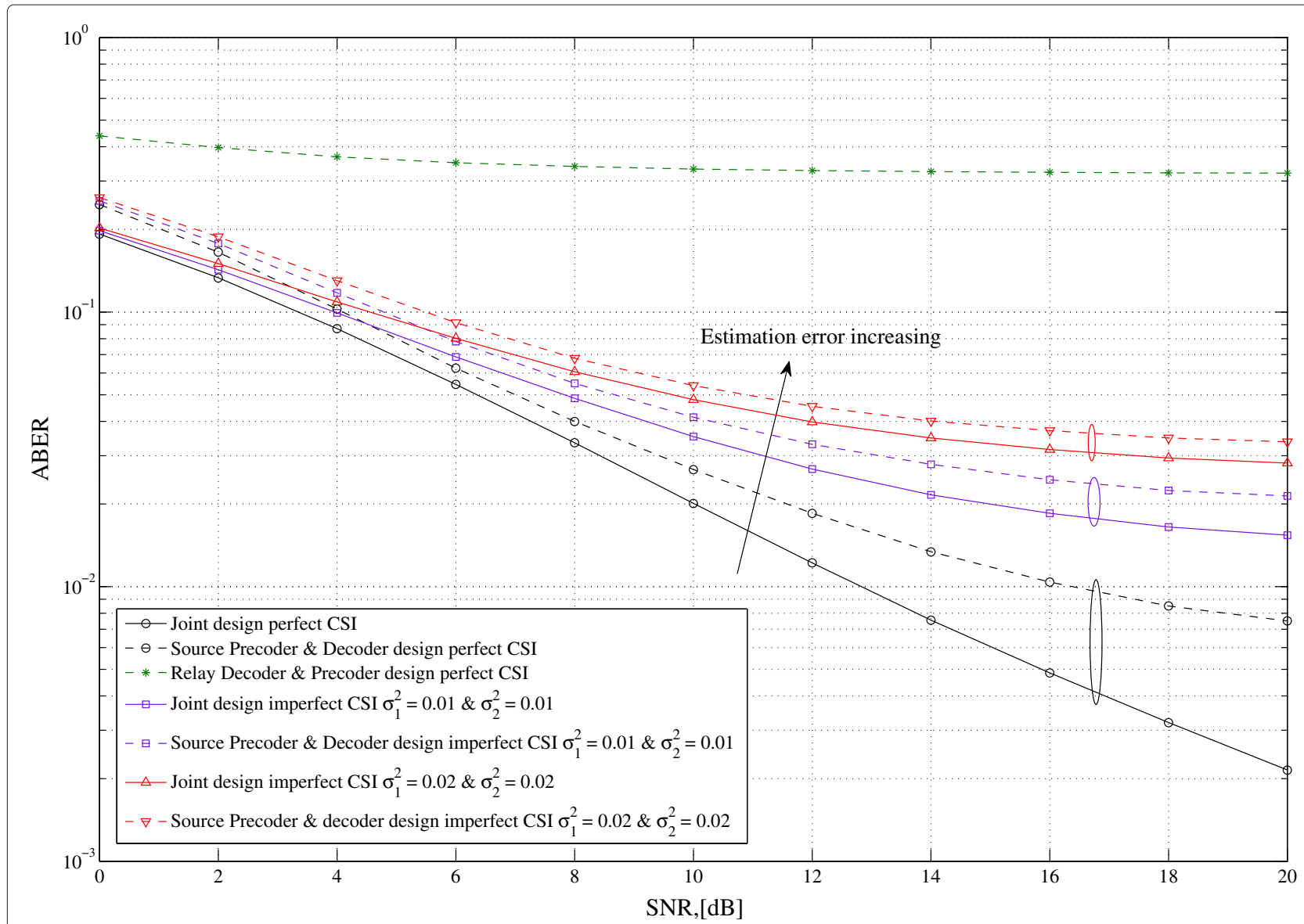

Figure 4 ABER versus transmit SNR: three different design schemes. Average BER variation with SNR $(\gamma)$ for three different design schemes $N=2, P_{T} / \sigma^{2}=8 \gamma$.

SNR. We consider each node to have two antennas $N=2, \epsilon=0.0001$, and the total transmit power $P_{T} / \sigma^{2}=8 \gamma$. Antenna correlation is not considered; it focuses only on the channel estimation errors. Two data streams are considered with three possible weight values $(0.25,0.50,0.75)$ for stream 1. AMSE reduces with the transmit SNR for every weight scenario. Also, when the weight parameter is higher, it further reduces the AMSE. This is therefore suitable to provide specific QoS requirements for multiple streams. Figure 7 shows a similar numerical analysis for the $\mathrm{BC}$ stage. The received signal at source 2 is used to find AMSE. In addition to the previous case, we assume the antenna correlation at the nodes, and it can be seen that the correlation has less impact on the performance compared to channel estimation errors.

Figure 8 shows the average number of iterations required for Algorithms 1 and 2 to converge with the number of antennas. We use $\mathbf{W}=\frac{1}{N} \mathbf{I}_{N}, \gamma=4 \mathrm{~dB}$, $\sigma_{1}=\sigma_{2}=0.02, \rho_{S 1}=\rho_{S 2}=\rho_{R}=0.5, \epsilon=0.0001$ and
$P_{T} / \sigma^{2}=4 N \gamma$. When the system has a higher number of antennas, more iterations are needed for Algorithm 1. These are realistic numbers, which can be used in practice with large MIMO systems. Algorithm 2 converges with a fewer number of iterations, and the variation with the initial point is extremely low.

\section{Conclusions}

We proposed a robust joint precoder-decoder design scheme for the PNC-based MIMO two-way relaying system when the perfect CSI is not available. In this design, minimizing weighted MSE problems are considered at both MA and BC stages. In the MA stage, PNC mapping is considered at the relay node. During the $\mathrm{BC}$ stage, joint design was needed to assist the decoding of the sum of two symbols at source nodes. The joint design problems became non-convex, and we divided those into subproblems and solved them iteratively. Numerical results are presented by considering the BER performance, MSEwith different weight matrices, relay location, and the number of antennas. Our research demonstrated that 


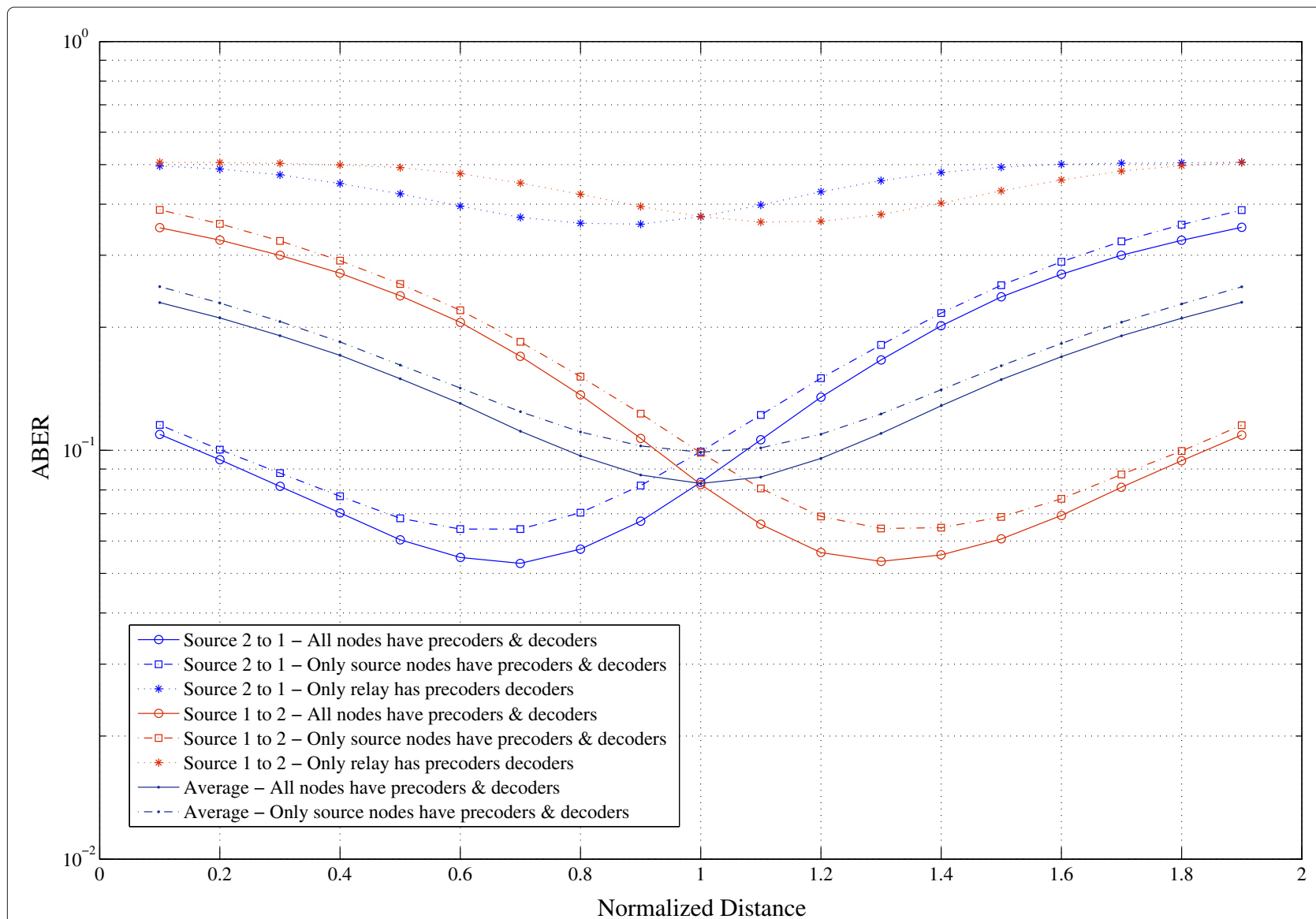

Figure 5 ABER versus normalized distance $(\boldsymbol{d})$. Average BER variation with normalized distance (d) for three different design $s c h e m e s . ~ N=2$, $\gamma=4 \mathrm{~dB}$, and $P_{T} / \sigma^{2}=32 \mathrm{~dB}$.

the joint precoder-decoder design performs better than the other schemes. The weight matrix can be used to provide QoS requirements of multiple data streams in MIMO two-way channels. The midpoint is seen to be the best location for the relay node to assist two-way communication. The proposed algorithm converges in a fewer iterations, and the number of iterations increases slowly with the number of antennas. These findings can help to have less complex PNC operation, improve the error performances, and mitigate the half-duplex issue of cooperative relays.

\section{Appendix}

\section{Existence of a global solution}

Problem 1

As in ([34], p. 133, Sec. 4.1.3), an optimization problem with several variables can always be minimized by initially minimizing some of the variables and then minimizing the remaining ones. Therefore, the optimization problem given in (19) can be reformulated as follows:

$$
\begin{aligned}
& \min _{\mathbf{F}_{1}, \mathbf{F}_{2}, \operatorname{Tr}\left(\mathbf{F}_{1} \mathbf{F}_{1}^{H}\right)+\operatorname{Tr}\left(\mathbf{F}_{2} \mathbf{F}_{2}^{H}\right) \leq P_{T}} \min _{\mathbf{G}\left[\mathbf{F}_{1}, \mathbf{F}_{2}\right]} \\
& \times \mathcal{E}_{\mathbf{E}}\left\{\mathbf{W M S E}_{m}\left[\mathbf{F}_{1}, \mathbf{F}_{2}, \mathbf{G}\left[\mathbf{F}_{1}, \mathbf{F}_{2}\right]\right]\right\},
\end{aligned}
$$

where $\mathbf{G}=\mathbf{G}\left[\mathbf{F}_{1}, \mathbf{F}_{2}\right]$ is a function of $\mathbf{F}_{1}$ and $\mathbf{F}_{2}$. Inner optimization in problem (36) has no constraints, and the solution for $\mathbf{G}\left[\mathbf{F}_{1}, \mathbf{F}_{2}\right]$ is given as follows:

$$
\begin{aligned}
\mathbf{G}\left[\mathbf{F}_{1}, \mathbf{F}_{2}\right]= & \left(\mathbf{F}_{1}^{H} \overline{\mathbf{H}}_{m 1}^{H}+\mathbf{F}_{2}^{H} \overline{\mathbf{H}}_{m 2}^{H}\right) \\
& \times\left(\overline{\mathbf{H}}_{m 1} \mathbf{F}_{1} \mathbf{F}_{1}^{H} \overline{\mathbf{H}}_{m 1}^{H}+\overline{\mathbf{H}}_{m 2} \mathbf{F}_{2} \mathbf{F}_{2}^{H} \overline{\mathbf{H}}_{m 2}^{H}\right. \\
& \left.+\operatorname{Tr}\left(\sigma_{1}^{2} \mathbf{Q}_{1}+\sigma_{2}^{2} \mathbf{Q}_{2}\right) \mathbf{R}_{r}+\sigma^{2} \mathbf{I}_{N}\right)^{-1} .
\end{aligned}
$$

Then, we can replace $\mathbf{G}$ with that, and the final objective function becomes variables of $\mathbf{F}_{1}$ and $\mathbf{F}_{2}$ :

$$
\min _{\mathbf{F}_{1}, \mathbf{F}_{2}, \operatorname{Tr}\left(\mathbf{F}_{1} \mathbf{F}_{1}^{H}\right)+\operatorname{Tr}\left(\mathbf{F}_{2} \mathbf{F}_{2}^{H}\right) \leq P_{T}} \mathcal{E}_{\mathbf{E}}\left\{\mathrm{WMSE}_{m}\left[\mathbf{F}_{1}, \mathbf{F}_{2}\right]\right\}
$$




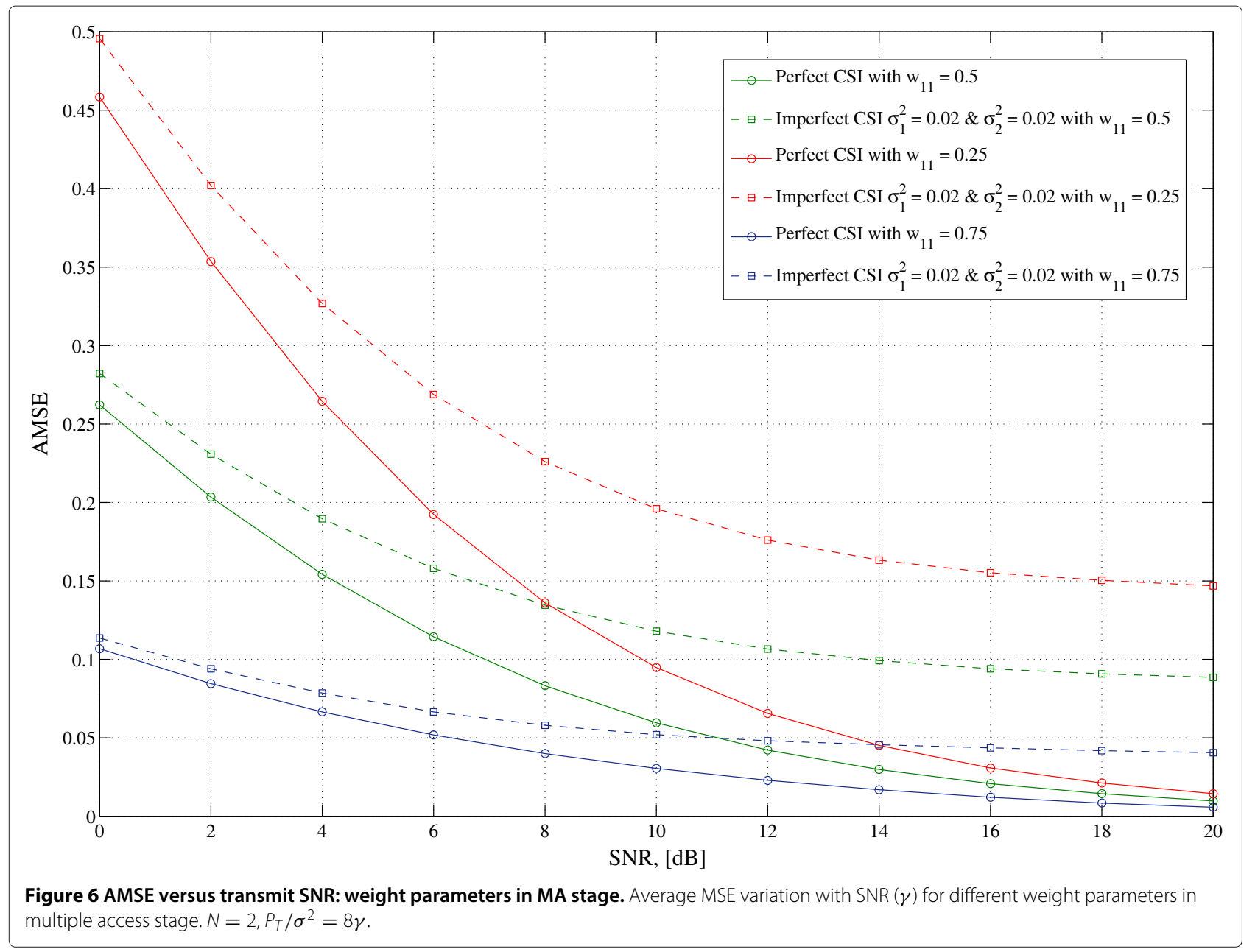

where

$$
\begin{aligned}
& \mathcal{E}_{\mathbf{E}}\left\{\mathrm{WMSE}_{m}\left[\mathbf{F}_{1}, \mathbf{F}_{2}\right]\right\} \\
&=\sum_{i=1}^{2} \operatorname{Tr}\left(\mathbf{W G}\left[\mathbf{F}_{1}, \mathbf{F}_{2}\right] \overline{\mathbf{H}}_{m i} \mathbf{F}_{i} \mathbf{F}_{i}^{H} \overline{\mathbf{H}}_{m i}^{H} \mathbf{G}\left[\mathbf{F}_{1}, \mathbf{F}_{2}\right]^{H}\right. \\
& \quad-\mathbf{W G}\left[\mathbf{F}_{1}, \mathbf{F}_{2}\right] \overline{\mathbf{H}}_{m i} \mathbf{F}_{i} \\
&\left.\quad-\mathbf{W} \mathbf{F}_{i}^{H} \overline{\mathbf{H}}_{m i}^{H} \mathbf{G}\left[\mathbf{F}_{1}, \mathbf{F}_{2}\right]^{H}+\mathbf{W}\right) \\
&+\operatorname{Tr}\left(\sigma^{2} \mathbf{W} \mathbf{G} \mathbf{G}^{H}\right) \\
&+\sum_{i=1}^{2} \sigma_{i}^{2} \operatorname{Tr}\left(\left(\mathbf{I}_{N}+\sigma_{i}^{2} \mathbf{R}_{i}^{-1}\right)^{-1} \mathbf{F}_{i} \mathbf{F}_{i}^{H}\right) \\
& \times \operatorname{Tr}\left(\mathbf{R}_{r} \mathbf{G}\left[\mathbf{F}_{1}, \mathbf{F}_{2}\right]^{H} \mathbf{W} \mathbf{G}\left[\mathbf{F}_{1}, \mathbf{F}_{2}\right]\right) .
\end{aligned}
$$

We can see that the feasible set of the optimization problem (38) is closed and bounded as in ([34], p. 30, Sec. 2.2.3). Since this is closed and bounded, the feasible set is compact according to ([35], p. 653, A.6 (g)). Additionally, the objective function (39) is continuous at all points of the feasible set. Therefore, according to the theorem in
([35], p. 654, A.8), there exists a global minimum for the problem (38). Finally, we can conclude that in ([34], p. 130, Sec. 4.1.3), there exists a global minimum for the original problem.

\section{Problem 2}

A similar procedure is valid to show the existence of a global minimum for Problem 2. However, for the sake of completeness, we describe it as given in this section. Optimization problem in (24) can be reformulated as follows ([34], p. 133, Sec. 4.1.3):

$$
\begin{gathered}
\min _{\mathbf{F}_{r}, \operatorname{Tr}\left(\mathbf{F}_{r} \mathbf{F}_{r}^{H}\right) \leq P_{T} / 2} \min _{\mathbf{G}_{1}\left[\mathbf{F}_{r}\right], \mathbf{G}_{2}\left[\mathbf{F}_{r}\right]} \\
\times \frac{1}{2} \mathcal{E}_{\mathbf{E}}\left\{\mathrm{WMSE}_{1}\left[\mathbf{F}_{r}, \mathbf{G}_{1}\left[\mathbf{F}_{r}\right]\right]\right\} \\
+\frac{1}{2} \mathcal{E}_{\mathbf{E}}\left\{\mathrm{WMSE}_{2}\left[\mathbf{F}_{r}, \mathbf{G}_{2}\left[\mathbf{F}_{r}\right]\right]\right\}
\end{gathered}
$$

where both $\mathbf{G}_{1}=\mathbf{G}_{1}\left[\mathbf{F}_{r}\right]$, and $\mathbf{G}_{2}=\mathbf{G}_{2}\left[\mathbf{F}_{r}\right]$ are functions of variable $\mathbf{F}_{r}$. Inner optimization problem has two parts, 


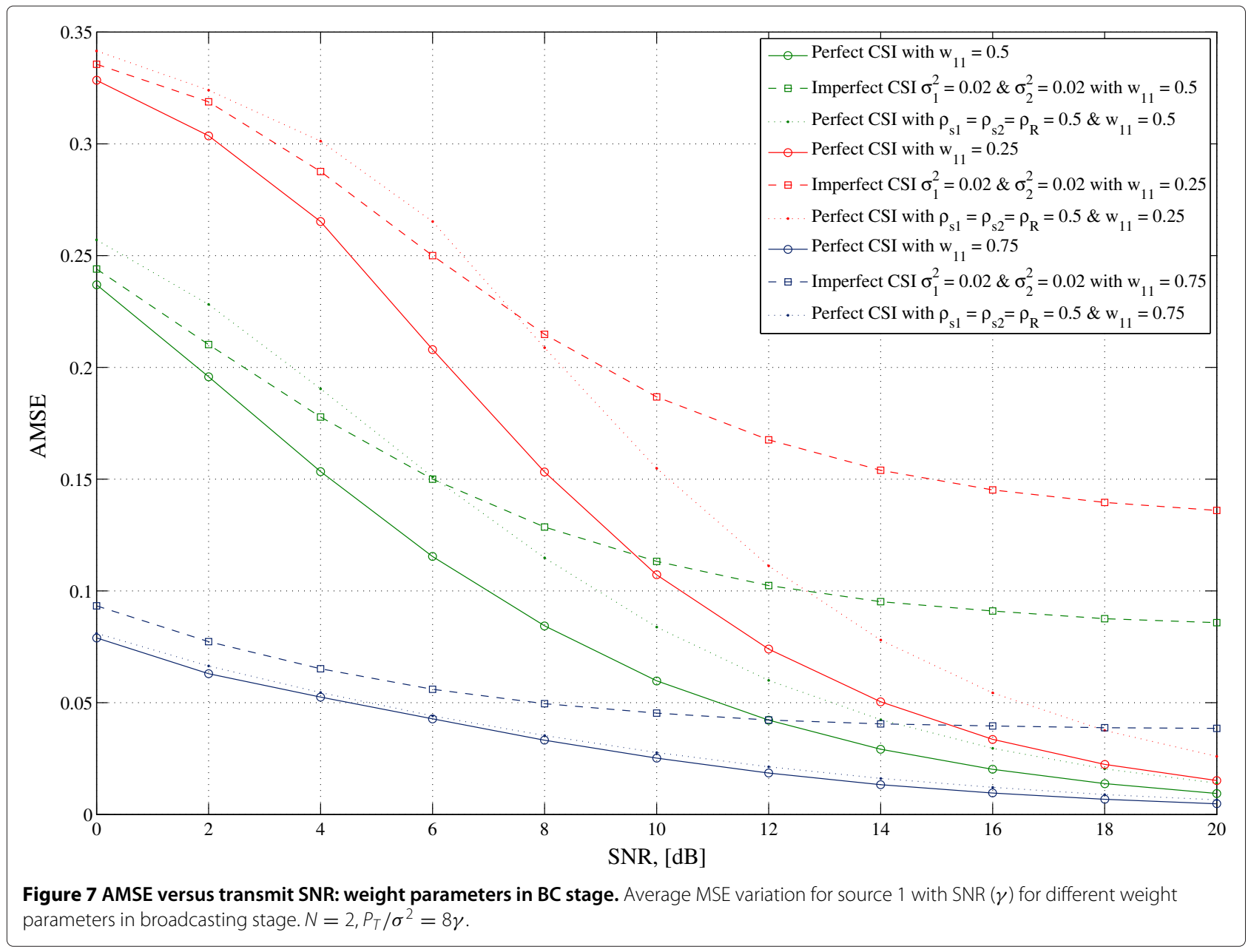

which are independent with $\mathbf{F}_{r}$ which is fixed. Also, the problem has no constraints. Therefore, the problem can be solved by taking derivatives and making those equal to zero.

$$
\begin{aligned}
\mathbf{G}_{i}\left[\mathbf{F}_{r}\right]= & \left(2 \mathbf{F}_{r}^{H} \overline{\mathbf{H}}_{m i}^{*}\right) \\
& \times\left(2 \overline{\mathbf{H}}_{m i}^{T} \mathbf{F}_{r} \mathbf{F}_{r}^{H} \overline{\mathbf{H}}_{m i}^{*}+2 \sigma_{i}^{2} \operatorname{Tr}(\mathbf{K})\right. \\
& \left.\times\left(\mathbf{I}_{N}+\sigma_{i}^{2} \mathbf{R}_{i}^{-1}\right)^{-1}+\sigma^{2} \mathbf{I}_{N}\right)^{-1} \quad i=1,2 .
\end{aligned}
$$

Next, we can replace $\mathbf{G}_{1}\left[\mathbf{F}_{r}\right]$ and $\mathbf{G}_{2}\left[\mathbf{F}_{r}\right]$ with abovementioned solutions to find the following optimization problem:

$$
\min _{\mathbf{F}_{r}, \operatorname{Tr}\left(\mathbf{F}_{r} \mathbf{F}_{r}^{H}\right) \leq P_{T} / 2} \frac{1}{2} \mathcal{E}_{\mathbf{E}}\left\{\mathrm{WMSE}_{1}\left[\mathbf{F}_{r}\right]\right\}+\frac{1}{2} \mathcal{E}_{\mathbf{E}}\left\{\mathrm{WMSE}_{2}\left[\mathbf{F}_{r}\right]\right\}
$$

where

$$
\begin{aligned}
\mathcal{E}_{\mathbf{E}}\left\{\mathrm{WMSE}_{i}\left[\mathbf{F}_{r}\right]\right\} \\
=2 \operatorname{Tr}\left(\mathbf{W G}_{i}\left[\mathbf{F}_{r}\right] \overline{\mathbf{H}}_{m i}^{T} \mathbf{F}_{r} \mathbf{F}_{r}^{H} \overline{\mathbf{H}}_{m i}^{*} \mathbf{G}_{i}\left[\mathbf{F}_{r}\right]^{H}\right. \\
\left.\quad \quad-\mathbf{W G}_{i}\left[\mathbf{F}_{r}\right] \overline{\mathbf{H}}_{m i}^{T} \mathbf{F}_{r}-\mathbf{W} \mathbf{F}_{r}^{H} \overline{\mathbf{H}}_{m i}^{*} \mathbf{G}_{i}\left[\mathbf{F}_{r}\right]^{H}+\mathbf{W}\right) \\
\quad+2 \sigma_{i}^{2} \operatorname{Tr}\left(\left(\mathbf{I}_{N}+\sigma_{i}^{2} \mathbf{R}_{i}^{-1}\right)^{-1} \mathbf{G}_{i}\left[\mathbf{F}_{r}\right]^{H} \mathbf{W G}_{i}\left[\mathbf{F}_{r}\right]^{H}\right) \\
\quad \times \operatorname{Tr}\left(\mathbf{R}_{r} \mathbf{F}_{r} \mathbf{F}_{r}^{H}\right)+\operatorname{Tr}\left(\sigma^{2} \mathbf{W G}_{i}\left[\mathbf{F}_{r}\right] \mathbf{G}_{i}\left[\mathbf{F}_{r}\right]^{H}\right) \quad i=1,2 .
\end{aligned}
$$

Similar to the previous case, we can see that the feasible set of optimization problem (42) is closed and bounded as in ([34], p. 30, Sec. 2.2.3), and it becomes compact according to ([35], p. 653, A.6 (g)). The objective function (43) is continuous at all points of the feasible set. Therefore, according to the theorem ([35], p. 654, A.8), there exists a global minimum for the problem (42). Finally, we can conclude that by ([34], p. 130, Sec. 4.1.3), there exists a global minimum for the original problem. 


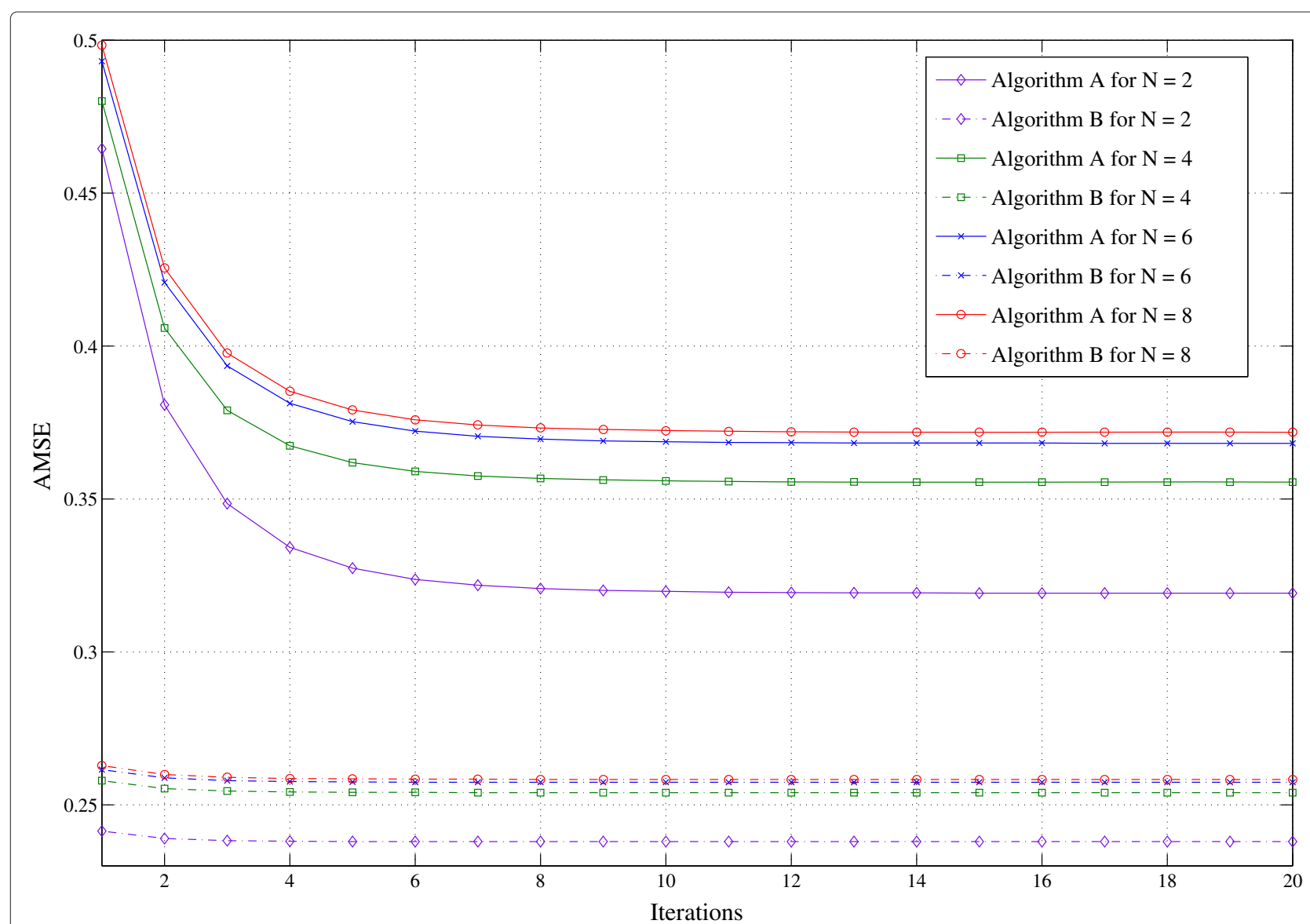

Figure 8 AMSE versus number of iteration. Average number of iteration to converge with the number of antennas. $\gamma=4 \mathrm{~dB}$ and $P_{T} / \sigma^{2}=4 \mathrm{~N} \gamma$.

\section{Competing interests}

The authors declare that they have no competing interests.

\section{Acknowledgements}

The authors would like to acknowledge the support of the European Commission by partially funding this work, under project FP7-ICT-2009-4-247733-EARTH, Finnish Funding Agency for Technology and Innovation (Tekes), Renesas Mobile, Nokia Siemens Networks, Elektrobit.

\section{Received: 1 January 2013 Accepted: 16 May 2013}

Published: 26 May 2013

\section{References}

1. JN Laneman, GW Wornell, Energy-efficient antenna sharing and relaying for wireless networks. Proc. IEEE, Wireless Commun. Networ. Confe. 1, 7-12 (2000)

2. G Scutari, S Barbarossa, D Ludovici, in Proceedings of 4th IEEE Workshop on Signal Processing Advances in Wireless Communications SPAWC 2003 Rome. Cooperation diversity in multihop wireless networks using opportunistic driven multiple access (IEEE Piscataway, 2003), pp. 170-174

3. Munoz O, A Agustin, J Vidal, in International Zurich Seminar on Communications (IZS), Zurich. Cellular capacity gains of cooperative MIMO transmission in the downlink (Piscataway, 2004), pp. 22-26

4. S Katti, H Rahul, W Hu, D Katabi, M Médard, J Crowcroft, XORs in the air: practical wireless network coding. IEEE/ACM Trans. Netw. (TON). 16(3), 497-510 (2008)

5. P Larsson, N Johansson, K Sunell, Coded bi-directional relaying. Proc. IEEE 63rd Veh. Technol. Conf. 2, 851-855 (2006)
6. B Rankov, A Wittneben, Spectral efficient protocols for half-duplex fading relay channels. IEEE J. Sel. Areas Commun. 25(2), 379-389 (2007)

7. S Zhang, SC Liew, PP Lam, in Proceedings of the 12th Annual International Conference on Mobile Computing and Networking (MobiCom '06). Hot topic: physical-layer network coding (ACM New York, 2006), p. 365

8. S Zhang, SC Liew, L Lu, in Proceedings of IEEE Global Telecommunications Conference (GLOBECOM), LOS Angeles. Physical layer network coding schemes over finite and infinite fields (IEEE Piscataway, 2008), pp. 1-6

9. P Popovski, H Yomo, Bi-directional amplification of throughput in a wireless multi-hop network. Proc. IEEE, 63rd Vehi. Tech. Conf. 2, 588-593 (2006). VTC 2006-Spring

10. B Nazer, M Gastpar, Compute-and-forward: Harnessing interference through structured codes. IEEE Trans. Infor. Theo. 57(10), 6463-6486 (2011)

11. IE Telatar, Capacity of multi-antenna Gaussian channels. Euro. Trans. Telecommun. 10(6), 585-596 (1999)

12. JH Winters, I Salz, RD Gitlin, The impact of antenna diversity on the capacity of wireless communication systems. IEEE Trans. Commun. 42(2), 1740-1751 (1994)

13. MO Hasna, MS Alouini, End-to-end performance of transmission systems with relays over Rayleigh-fading channels. IEEE Trans. Wireless Commun. 2(6), 1126-1131 (2004)

14. GK Karagiannidis, TA Tsiftsis, RK Mallik, Bounds for multihop relayed communications in Nakagami-m fading. IEEE Trans. Commun. 54, 18-22 (2006)

15. SXu, Y Hua, in Proceedings of IEEE International Conference on Acoustics Speech and Signal Processing (ICASSP), Texas. Source-relay optimization for a two-way MIMO relay system (IEEE Piscataway, 2010), pp. 3038-3041

16. S Kim, J Chun, in Proceedings of IEEE Wireless Communications and Networking Conference (WCNC), Las Vegas. Network coding with linear 
MIMO pre-equalizer using modulo in two-way channel (IEEE Piscataway, 2008), pp. 517-521

17. H Yang, K Lee, J Chun, in Proceedings of IEEE International Conference on Communications (ICC) 2007 Glasgow. Zero-forcing based two-phase relaying (IEEE Piscataway, 2007), pp. 5224-5228

18. S Zhang, S Liew, in Proceedings of IEEE Wireless Communications and Networking Conference (WCNC), Sydney. Physical layer network coding with multiple antennas (IEEE Piscataway, 2010), pp. 1-6

19. LKS Jayasinghe, N Rajatheva, M Latva-aho, in Proceedings of IEEE Wireless Communications and Networking Conference (WCNC), Paris. Energy efficient MIMO two-way relay system with physical layer network coding (IEEE Piscataway, 2012), pp. 1-5

20. W Guan, H Luo, Joint MMSE transceiver design in non-regenerative MIMO relay systems. IEEE Commun. Lett. 12(7), 517-519 (2008)

21. KJ Lee, KW Lee, H Sung, I Lee, in Proceedings of IEEE 69th VTC Spring 2009 Barcelona. Sum-rate maximization for two-way MIMO amplify-and-forward relaying systems (IEEE Piscataway, 2009), pp. 1-5

22. S Xu, Y Hua, Optimal design of spatial source-and-relay matrices for a non-regenerative two-way MIMO relay system. IEEE Trans. Wireless Commun. 10(5), 1645-1655 (2011)

23. R Wang, M Tao, Joint source and relay precoding designs for MIMO two-way relaying based on MSE criterion. Tran. Sign. Proc. 60(3), 1352-1365 (2012)

24. B Hassibi, B Hochwald, How much training is needed in multiple-antenna wireless links?. IEEE Trans. Infor. Theo. 49(4), 951-963 (2003)

25. C Xing, $\mathrm{S} M a, Y \mathrm{Wu}$, Robust joint design of linear relay precoder and destination equalizer for dual-hop amplify-and-forward MIMO relay systems. IEEE Trans. Sig. Proc. 58(4), 2273-2283 (2010)

26. CXing, Z Fei, Y Wu, S Ma, J Kuang, in Proceedings of IEEE ICSPCC 2011 Shaanxi. Robust transceiver design for AF MIMO relay systems with column correlations (IEEE Piscataway, 2011), pp. 1-6

27. M Ding, S Blostein, MIMO minimum total MSE transceiver design with imperfect CSI at both ends. IEEE Trans. Sig. Proc. 57(3), 1141-1150 (2009)

28. Z Ding, KK Leung, Impact of imperfect channel state information on bi-directional communications with relay selection. IEEE Trans. Sig. Proc. 59(11), 5657-5662 (2011)

29. S Thoen, L Van der Perre, B Gyselinckx, M Engels, Performance analysis of combined transmit-SC/receive-MRC. IEEE Trans. Commun. 49, 5-8 (2001)

30. HA Suraweera, TA Tsiftsis, GK Karagiannidis, M Faulkner, in Proceedings of IEEE GLOBECOM 2009 Hawaii. Effect of feedback delay on downlink amplify-and-forward relaying with beamforming (IEEE Piscataway, 2009), pp. 1-6

31. G Amarasuriya, C Tellambura, M Ardakani, in Proceedings of IEEE GLOBECOM 2010 Miami. Feedback delay effect on dual-hop MIMO AF relaying with antenna selection (IEEE Piscataway, 2010), pp. 1-5

32. T Koike-Akino, P Popovski, V Tarokh, Optimized constellations for two-way wireless relaying with physical network coding. IEEE J. Sel. Areas Commun. 27(5), 773-787 (2009)

33. A Hjorungnes, D Gesbert, Complex-valued matrix differentiation: techniques and key results. IEEE Trans. Sign. Proces. 55(6), 2740-2746 (2007)

34. S Boyd, L Vandenberghe, Convex Optimization. (Cambridge University Press, Cambridge, 2004)

35. DP Bertsekas, Nonlinear Programming. (Athena Scientific, Nashua, 1999)

doi:10.1186/1687-1499-2013-137

Cite this article as: Saliya Jayasinghe et al:: Robust precoder-decoder design for physical layer network coding-based MIMO two-way relaying system. EURASIP Journal on Wireless Communications and Networking 2013 2013:137. 